\title{
HEAT PIPE EFFECT IN POROUS MEDIUM
}

\author{
by
}

\section{Martin Joseph}

A thesis submitted in partial fulfillment

of the requirements for the degree of

\section{Master of Science in Engineering \\ in}

\section{Civil and Environmental Engineering}

\author{
Department of Civil and Environmental Engineering \\ University of Nevada, Las Vegas
}

December, 1992

\begin{abstract}
DISCLAIMER
This report was prepared as an account of work sponsored by an agency of the United States Government. Neither the United States Government nor any agency thereof, nor any of their employees, makes any warranty, express or implied, or assumes any legal liability or responsibility for the accuracy, completeness, or usefulness of any information, apparatus, product, or process disclosed, or represents that its use would not infringe privately owned rights. Reference herein to any specific commercial product, process, or service by trade name, trademark, manufacturer, or otherwise does not necessarily constitute or imply its endorsement, recommendation, or favoring by the United States Government or any agency thereof. The views and opinions of authors expressed herein do not necessarily state or reflect those of the United States Government or any agency thereof.
\end{abstract}


The thesis of Mr. Iartin Jusepin tor the degree of Mlaster of Science in Engineering in Civii and Environmental Engineering is approved.

Chairperson. Protessor James Carale. Ph.D

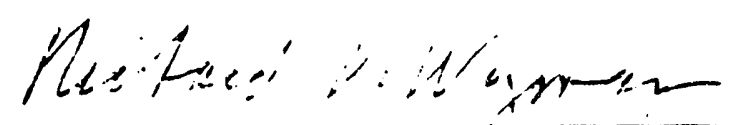

Examining Committee Wember, Photessor Richard Wyman. Ph.D

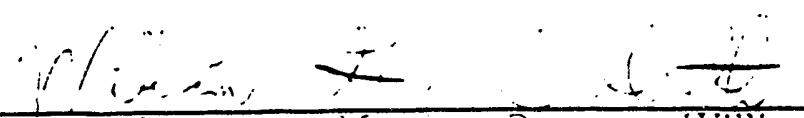

Examining Committee :Iember. Protessor William Culbreth, Ph.D

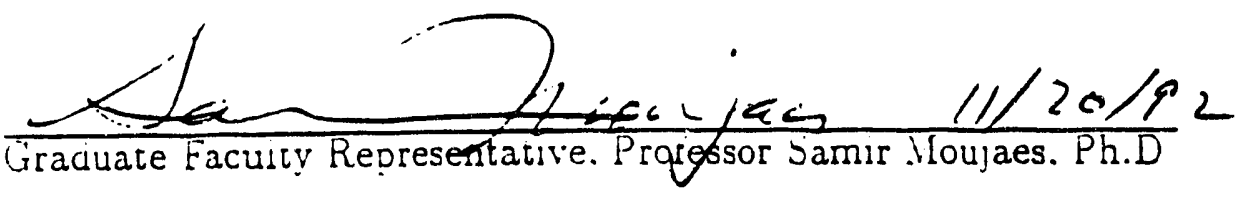

Tiaduate Dean. Protessor Ronald IV. Smitn. Ph.D

$$
\text { Eniversity of Nerada. Las legas }
$$

Dec:emiver. 1992: 


\begin{abstract}
In this thesis. a parametric study of the thermal and hydrologic characteristics of the fractured porous tuffs at Yucca Mountain, Nevada was conducted. The effects of different fracture and matrix properties including permeability, thermal conductivity, specific heat, porosity. and tortuosity on heat pipe performance in the vicinity of the waste package were observed. Computer simulations were carried out using TOUGH code on a Cray YMP-2 supercomputer.

None of the fracture parameters affected the heat pipe performance except the mobility of the liquid in the fracture. Matrix permeability and thermal conductivity were found to have significant effect on the heat pipe performance. The effect of mass injection was studied for liquid water and air injected at the fracture boundary. A high rate of mass injection was required to produce any effect on the heat pipe. The fracture-matrix equilibrium is influenced by the matrix permeability and the matrix thermal conductivity.
\end{abstract}




\section{Contents}

Abstract $\ldots \ldots \ldots \ldots \ldots \ldots \ldots \ldots \ldots \ldots \ldots \ldots \ldots \ldots \ldots$ iii

Acknowledgments $\ldots \ldots \ldots \ldots \ldots \ldots \ldots \ldots \ldots$ iv

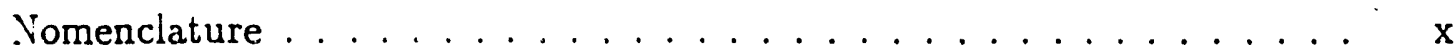

1 INTRODUCTION 1

2 LITERATURE REVIEW

3 DESCRIPTION AND USE OF THE TOUGH CODE 9

3.1 GOVERNING FLOW EQUATIONS $\ldots \ldots \ldots \ldots \ldots$

4 DESCRIPTION OF MODELING 16

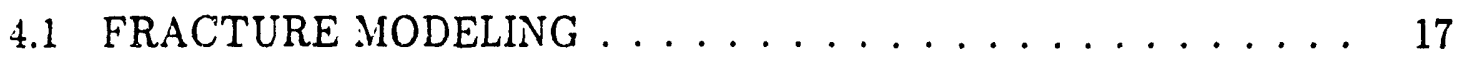

4.1.1 RELATIVE PERMEABILITY \& CAPILLARY PRESSURE . 18

4.1.2 FRACTURE PERMEABILITY . . . . . . . . . . 21

4.1.3 FRACTURE THERMAL CONDUCTIVITY . . . . . . . 26

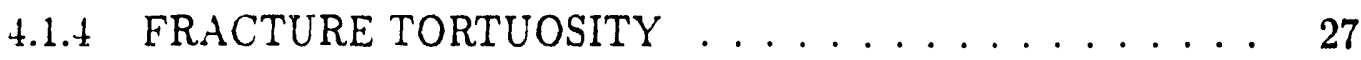

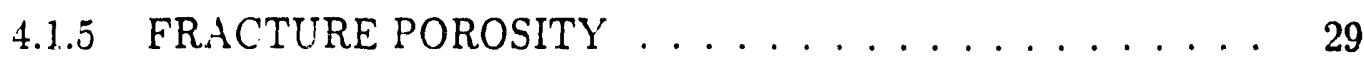

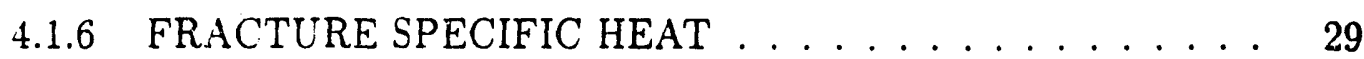

4.2 FRACTURE MODELING RESULTS . . . . . . . . . . . 30

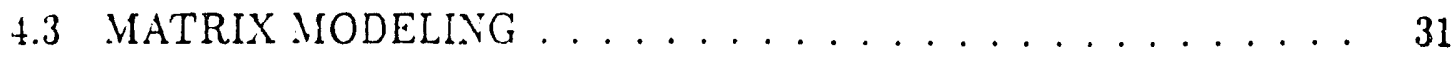

4.3 .1 CHARACTERISTIC FUNCTIONS . . . . . . . . . 31

4.3 .2 . MATRIX PERMEABILITY . . . . . . . . . . . 33

4.3 .3 MATRIX THERMAL CONDUCTIVITY . . . . . . . 38 


\section{List of Figures}

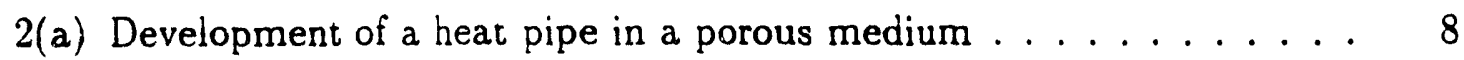

4 (a) Geometry of the system modeled . . . . . . . . . . . . 54

4(b) Temperature profiles for different permeabilities at 51 days . . . . . 55

4(c) Liquid saturation profile for $k_{m}=3.2 \times 10^{-18} \mathrm{~m}^{2}$ at 51 days $\ldots \ldots 56$

4(d). Liquid saturation profile for $k_{m}=3.2 \times 10^{-15} \mathrm{~m}^{2}$ at 51 days $\ldots . .57$

4(e) Temperature profiles for different permeabilities at 1 year . . . . . 58

4(f) Gas pressure profile at 1 year for $k_{m}=3.2 \times 10^{-18} \mathrm{~m}^{2} \ldots \ldots \ldots 59$

4(g) Gas pressure profile $t 1$ year for $k_{m}=3.2 \times 10^{-15} \mathrm{~m}^{2} \ldots \ldots \ldots 60$

4(h) Capillary pressure profile at 1 year for $k_{m}=3.2 \times 10^{-18} \mathrm{~m}^{2} \ldots \ldots 61$

4(i) Capillary pressure profile at 1 year for $k_{m}=3.2 \times 10^{-15} \mathrm{~m}^{2} \ldots \ldots 62$

$4(\mathrm{j})$ Temperature profiles at 1 year for different therrnal conductivities . . 63

4(k) Capillary pressure proniles at 1 year for different thermal conductivities 64

4 (l) Temperature profiles at 1 year for different specific heat . . . . . . 65

5(a) Temperature profiles at 1 year for different water injection rates $\ldots 73$

5(b) Temperature profiles at 1 year for different air injection rates . . . . 74 


\section{List of Tables}

4.1 Parametric values for the fracture linear capillary pressure function . 19

4.2 Parametric values for the fracture linear relative permeability function 20

4.3 Fracture parameters and their range . . . . . . . . . . . . 21

4.4 Temperature distributions for different fracture permeabilities for the immobile case . . . . . . . . . . . . . . . . 22

4.5 Temperature distributions for different fracture permeabilities for the mobile case . . . . . . . . . . . . . . . . 24

4.6 Temperature distributions for different fracture permeabilities for Grant's

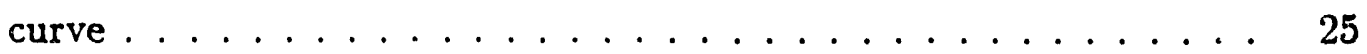

4.7 Temperature distributions for different fracture thermal conductivities after 51 days . . . . . . . . . . . . . . . . 27

4.8 Temperature distributions for different fracture tortuosities . . . . . 28

4.9 Temperature distributions for different fracture porosities . . . . . . 29

4.10 Temperature distributions for different heat capacities . . . . . . . 30

4.11 Matrix parameters and their range ............. . . 31

4.12 Parametric values for the Sandia functions . . . . . . . . . . 33

4.13 Numerical values of different matrix permeabilities . . . . . . . . . 34

4.14 Numerical values of different matrix thermal conductivities . . . . . 38

4.15 Numerical values of different matrix tortuosities . . . . . . . . . 40

4.16 Temperature distributions for different fracture tortuosities . . . . . . 40

4.17 Numerical values of different matrix porosities . . . . . . . . . . . 41

4.18 Temperature distributions for different matrix porosities . . . . . . 42 
4.19 Numerical values of different matrix heat capacities . . . . . . . . 42

4.20 Sensitivity of the heat pipe to different matrix parameters . . . . . 45

4.21 Sensitivity of the temperature to different matrix parameters . . . . 46

4.22 Nusselt numbers and their sensitivities at the heat pipe region . . . 48

5.1 Applied liquid fluxes and the corresponding velocities and pressure gradients at different regions . . . . . . . . . . . 67

5.2 Temperature distributions for different liquid fluxes . . . . . . . 67

5.3 Applied air fluxes and the corresponding velocities and pressure gradients at different regions . . . . . . . . . . . . . . . 69

5.4 Temperature distributions for different air fluxes . . . . . . . . . 70

5.5 Temperature distributions for different air withdrawal rates . . . . . 71

6.1 Comparison of the fracture-matrix equilibrium time . . . . . . . 77 


\section{NOMENCLATURE}

A $\quad \ldots$ area. $m^{2}$

$C_{p} \quad \ldots \quad$ specific heat at constant pressure, $J / k g^{\circ} \mathrm{C}$

$D_{v a} \quad \ldots$ binary diffusion coefficient. $\mathrm{m}^{2} / \mathrm{s}$

$D_{v a}^{\circ} \quad \ldots$ binary diffusion coefficient at standard conditions. $\mathrm{m}^{2} / \mathrm{s}$

$\vec{F}_{3}^{a} \quad \ldots \quad$ mass flux of air in gas phase. $\mathrm{kg} / \mathrm{s}$

$\vec{F}_{l}^{a} \quad \ldots$ mass flux of air in liquid phase. $\mathrm{kg} / \mathrm{s}$

$\overrightarrow{F_{3}^{w}} \quad \ldots \quad$ mass flux of water in gas phase. $\mathrm{kg} / \mathrm{s}$

$\overrightarrow{F_{l}^{w}} \quad \ldots$ mass flux of water in liquid phase, $\mathrm{kg} / \mathrm{s}$

$\vec{g} \quad \ldots$ gravitational acceleration vector. $\mathrm{m} / \mathrm{s}^{2}$

$k_{f} \quad \ldots \quad$ absolute fracture permeability, $m^{2}$

$k_{m} \quad \ldots \quad$ absolute matrix permeability, $m^{2}$

$k_{r l} \quad \ldots \quad$ liquid relative permeability

$k_{r g} \quad \ldots \quad$ gas relative permeability

K $\quad \ldots$ thermal conductivity, $W / m^{\circ} \mathrm{C}$

$K_{d r} \quad \ldots$ dry thermal conductivity, $W / m^{\circ} \mathrm{C}$

$K_{w e} \quad \ldots \quad$ saturated thermal conductivity, $W / m^{\circ} \mathrm{C}$

$V^{w} \quad \ldots \quad$ mass of water per unit volume

$W^{a} \quad \ldots \quad$ mass of air per unit volume

Nu $\quad \ldots \quad$ Nusselt number

$P_{g} \quad \ldots \quad$ gas pressure, $P_{1,}$

$P_{l} \quad \ldots \quad$ liquid pressure, $P_{i}$

$P_{v} \quad \ldots \quad$ vapor partial pressure. $P_{n}$ 


$$
\begin{aligned}
& P_{\text {sad }} \quad \ldots \quad \text { capiilary pressure. } P_{3} \\
& q_{a} \quad \ldots \quad \text { mass flux due to air. } \mathrm{kg} / \mathrm{s} \\
& q_{w} \quad \ldots \quad \text { mass dux due to water. } \mathrm{kg} / \mathrm{s} \\
& \text { Q ... total heat flow through overall section. W } \\
& Q_{f} \quad \ldots \quad \text { total heat flow through fracture. W } \\
& Q_{m} \quad \ldots \quad \text { total heac flow througn matrix. W } \\
& \text { Qed } \ldots \text { total heat conducted. W } \\
& \text { Qov } \quad \ldots \quad \text { total hear convected. W } \\
& \text { S } \quad \ldots \quad \text { sensitivity, } \% \\
& S_{l} \quad \ldots \quad \text { liquid saturation } \\
& S_{g} \quad \text { ‥ gas saturation } \\
& S_{l, f} \quad \ldots \quad \text { fracture liquid saturation } \\
& S_{l, m} \quad \ldots \quad \text { matrix liquid saturation } \\
& S_{\text {Ir.f }} \quad \ldots \quad \text { irreducible fracture liquid saturation } \\
& S_{\text {ir.m }} \quad \ldots \quad \text { irreducible matrix liquid saturation } \\
& S_{l s} \quad \ldots \text { scaled liquid saturation } \\
& t \quad \ldots \quad \text { time. } s \\
& \text { T } \quad \ldots \text { temperature. }{ }^{\circ} \mathrm{C} \\
& \mathrm{V} \quad \ldots \quad \text { volume. } \mathrm{m}^{3} \\
& V_{w, b} \quad \ldots \quad \text { liquid velocity at bore hole wall, } \mathrm{m} / \mathrm{s} \\
& V_{\text {a.b }} \quad \ldots \quad \text { air velocity at bore hole wall, } \mathrm{m} / \mathrm{s} \\
& x \quad \ldots \text { distance. } m \\
& \mathrm{X} \quad \ldots \quad \text { mass fraction }
\end{aligned}
$$




\section{Greek Symbols}

$\lambda$

$\mu$

$\nu$

$\rho$

$\tau$

$\phi$

\section{Subscripts}

a

b

f

g

1

m

v

w

$\mathrm{d} \mathbf{r}$

we

cd

$\mathrm{cv}$
.. Van Genuchten curve-fit parameter

... dynamic viscosity, $N-s / m^{2}$

... kinematic viscosity, $\mathrm{m}^{2} / \mathrm{s}$

... density, $\mathrm{kg} / \mathrm{s}$

... tortuosity factor

... porosity

... air

.. bore hole wail

... fracture

... gas

... liquid

... matrix

... vapor

... water

... dry

... wet

... conduction

... convection 


\section{Superscripts}

a

.. air

b

... heat

w

... water 


\section{Chapter 1}

\section{INTRODUCTION}

The C.S.Department of Energy (DOE) is investigating the feasibility of constructing and operating a high level nuclear waste repository in tuffaceous rocks occuring in the unsaturated zone at Yucca Mountain. Nevada. The thick tuff formations at Yucca Mountain, Nevada are located above the water table in partially saturated rock. The proposed repository is approximately 300 meters below the ground level and 150 meters above the water table in the Topopah Springs unit of the Yucca Mountain tuffs.

Site characterization involves the determination of the movement of the water through the rock. If a thermal source is introduced. a thermally driven flow will be established. The purpose of this thesis is to examine parametrically those factors that influence this thermaily driven flow in a fractured volcanic tuff.

A heat pipe is a highly efficient way of transfering thermal energy over a relatively short distance. The basic ingredients of a heat pipe are (i) a volatile fluid and (ii) a mechanism by which the liquid and the gas phases can flow in opposite directions. The phenomenon of a heat pipe is characterized by liquid-vapor counterflow and the muiti-phase regions. 
Heat pipes are being widely applied in engineered heat transfer systems. This heat pipe phenomenon is also important in high level nuclear waste disposal in a repository. The emplacement of a strong heat source. such as a high level was.e package, in a partially saturated permeable medium can give rise to the development of a heat pipe. Favorable conditions for the development of a heat pipe occur in a fractured medium with large fracture permeability and strong capillary effects in the rock matrix. The fracture provides a path for the movement of vapor away from the heat source. The vapor is condensed and the condensate is drawn through the rock matrix by capillary effects. Liquid condensate may also flow back towards the heat source through the fracture by gravitational force. The effective thermal conductivity of the rock will be very high due to the existence of a heat pipe.

The existence and the extent of the heat pipe is highly significant on the performance of the repository. The strength and the size of the heat pipe controls the temperature at the bore hole wall. If the heat pipe region extends to the heat source. the two phase region will also be extended to the canister. This will cause moisture to come into the vicinity of the container. This may eventuaily lead to the corrosion and the loss of integrity of the container. It also results in additional mass transport and therefore provides a pathway for contaminant transport.

Wathematicai modeling of the heat pipe involves the transport modeling of the water and heat. In this study, the type of heat and mass transfer resulting from the emplacement of nuclear waste canisters in a repository is studied in detail. The length of the heat pipe region. the point of condensation and the relative significance of convective heat transfer over conductive transfer or vice versa are examined in detail. 
The neat pipe effect depends on a variety of matrix and fracture properties and fluid properties. In this study, the fluid properties are assumed to be constant. Properties such as permeabiiity, thermal conductivity, heat caparity, and porosity of both matrix and fracture may be significant, but the effect of these parameters has to be explored fully. All these properties may change the point of condensation and the extent of the heat pipe region in a fractured medium. Many simulations were carried out using the TOUGH code for a range of values for the above properties to understand their exact influence on the heat pipe. These properties are tested for a wide range of values and the significance of each property on the performance of heat pipe is examined. The property values at which the heat pipe effect becomes the most predominant are thus determined.

The effect of mass injection on the heat pipe pe:formance is also examined in detail. Mass is injected in the form of liquid water and air. Finally, the factors that control fracture-matrix equilibrium and the time required to establish fracture-matrix equilibrium are examined. This has implications for the applicability of the effective continuum approach in modeling the fractured rock. 


\section{Chapter 2}

\section{LITERATURE REVIEW}

The mechanical heat pipe can be several thousand times as effective in transporting heat as the best metals [Eastman, 1968]. The heat pipe behavior in a fractured porous rock was well illustrated by [Pruess. 1985]. Fig.2(a) shows the schematic representation of a heat pipe development in a fractured porous medium due to the emplacement of nuclear a waste package. Heat enters into the matrix as soon as the canister is emplaced in the repository. This causes the vaporization of the liquid present in the rock. The vapor generated in this way flows through the fracture away from the canister because of the large fracture permeabil $i y$ and a slight pressure gradient. Condensation of the vapor takes place as it meets with the cooler region of the rock. Due to the large capillary effects in the matrix. the majority of the condensate is absorbed by the matrix. The difference in liquid saturation existing between the boiling point and the condensation point in the matrix causes the majority of the condensate to flow back towards the canister through the matrix. The iquid is then re-vaporized as it flows toward the canister. This cycle of liquid-vapor counter flow is repeated to develop a heat pipe region. 
The heat pipe phenomenon in porous media was analytically investigated and the governing transport equations are established for one dimensional heat pipe configurationiOgniewcz and Tien. 1979]. The effect of fractures was not considered in this study, but the solution for the one dimensional heat pipe demonstrated the effects of various fluid and porous media properties on the heat pipe periormance.

A one dimensional. steady state analysis of the heat and mass trasfer in a saturated porous media was carried out later [Udell. 1985]. In this analysis, the effects of capillarity, gravity forces. and phase change were included. The dry out heat flux was predicted and verified for the bottom heating orientation. It was found that. for a low permeability of $k<1 \times 10^{-14}$, the conductive heat transfer will be of the same order of magnitude as the convective heat transfer. The product of the heat flux and the two phase zone length was found to be constant for fixed fluid and media properties under conditions of high heat fluxes. The counter current flow allows for an extremely efficient heat transfer process similar to the operation of conventional heat pipes.

A quantitative model of vapor dominated geothermal reservoir as heat pipe in fractured rock was also given by [Pruess. 1985].

The emplacement of a strong heat source. such as a high level nuclear waste package, in a partially saturated permeable medium will give rise to the development of a heat pipe [Doughy and Pruess. 1988]. In this paper. a simplified version of the problem that has a steady state solution for a radial geometry where a semi-analytical solution was obtained. The parameters determining the heat pipe length and the question of whether the vicinity of the heat source dries up were also discussed. It was found that the extent of the heat pipe region can be much greater for radial geometry than for linear geometry because of 
the decrease in flow rate per unit area that occurs with radial distance. The characteristic curves for relative permeability and capillary pressure also have a strong influence on the extent of the heat pipe. Large permeability in fractures and strong capillary effects in the porous matrix provide conditions that are favorable for the development of the heat pipe.

Multi-phase heat and mass transfer in porous media was analyzed by [Fitch and Udell, 1985]. A one dimensional steady state model describing the heat and mass transfer within a porous medium was developed in this work. Heat and mass transfer in unsaturated porous materials was examined in detail for the application of the heat source in soil (Reddy, 1986]. This analysis showed that the diffusion into the soil by conduction is predominant in the early stages of heating. Extensive modeling studies cí multi-phase fluid and heat flow processes in nuclear waste isolation was conducted by [Pruess, 1989]. This model showed the important physical phenomena occuring in multiphase and nonisothermal flows. It was found that the expected temperature and moisture regime near the waste packages depends on the relative permeabilities of fractures. The effect of the liquid infiltration in an unsaturated, fractured, porous medium was studied by [Nitao et al., 1989]. The various physical processes involved in a one dimensional fracture dominated flow conditions in unsaturated porous medium were analyzed in this work.

The heat pipe effect in porous media was examined in detail by [Pruess et al., Part 1, 1990]. Modeling studies were done for simultaneous transport of heat. liquid water, vapor and air in partially saturated, fractured porous rock. The performance of the heat pipe was found to depend strongly on inobility of the liquid in the fracture. Thermohydrologic conditions in the vicinity of the waste packages were found to depend strongly on relative permeability and capillary pressure characteristics of fractures which are unknown. It was 
found that if the liquid held on the rough walls of drained fractures is assumed to be mobile. strong heat pipe effects can be predicted and the host rock will remain in multiphase conditions right up to the emplacement hole and formation temperatures will peak at the boiling temperature which is near $100^{\circ} \mathrm{C}$. Standard atmospheric pressure of $101 \mathrm{KPa}$ was observed at the bore hole wall so that elevation effect was not considered. In this work, the effect of gravity is neglected which is expected to be admissible due to the strength of capillary forces in the strong saturation gradients near the waste packages.

The idea of an effective continuum approximation for the modeling of fluid and heat flow in fractured porous media was introduced later by [Pruess et al., Part 2. 1990]. This approximation is based on the thermohydrologic behaviour observed in detailed simulations with explicit considerations of fracture effects in the previous paper. The crucial concept in the development of an effective continuum approximation is the notion of local thermodynamic equilibrium between rock matrix and fractures. The effective continuum approximation gave very accurate results in the case of a large matrix permeability(32.6 microdarcy), but poor results were obtained for a tight matrix(1.9 microdarcy). In general, an effective continuum approximation is applicable when sufficiently large space and time scales are considered.

Fracture and matrix hydrologic characteristics of tuffaceous materials from Yucca Mountain were given by [Peters et al.. 1984]. Most of the thermal and mechanical property values of the rock for the welded and non-welded tuffs of the G-tunnel of the Nevada Test site are taken from this report. These values are used to test the different matrix and fracture properties in this heat pipe study. 


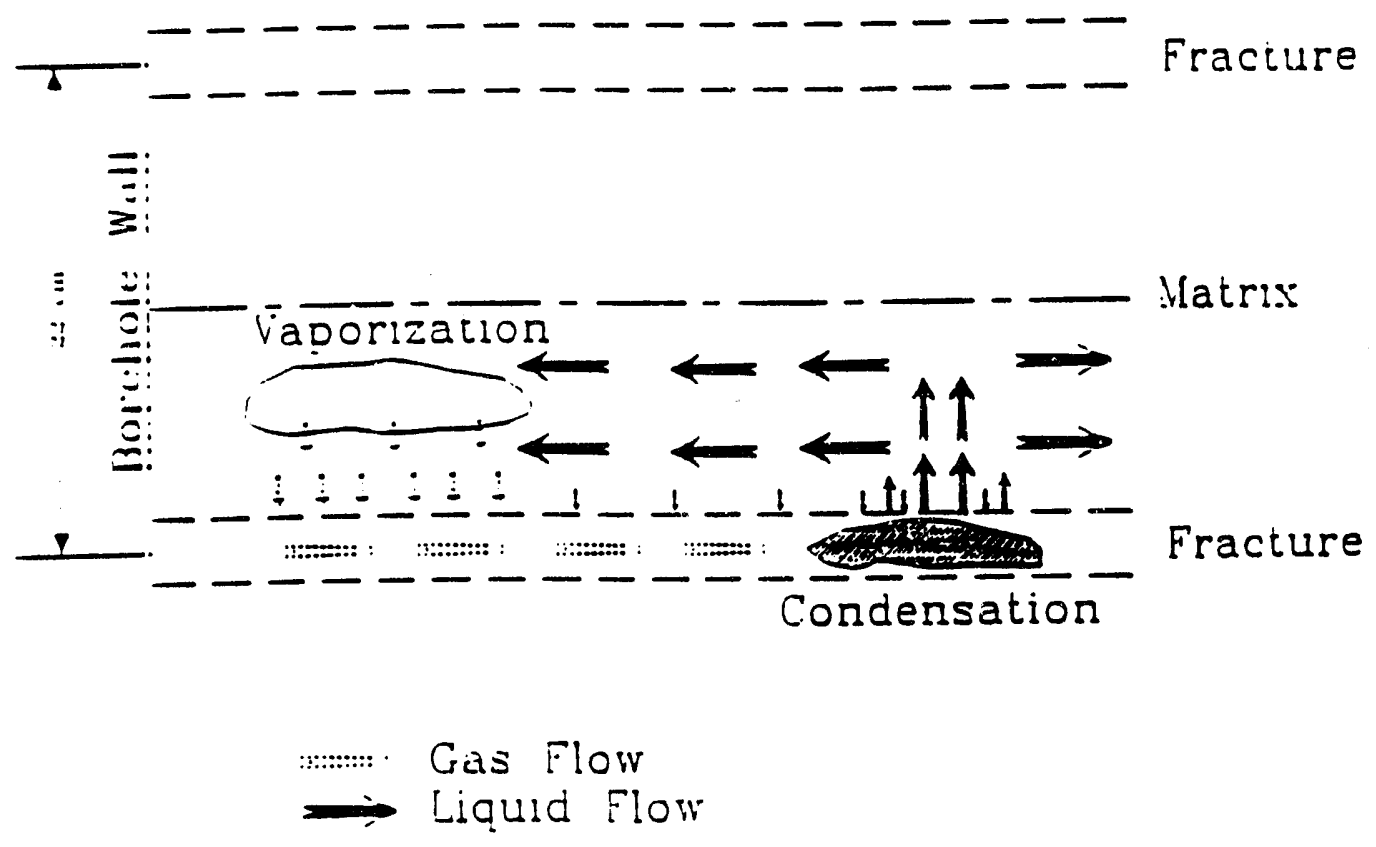

Figure 2(a): Development of a heat pipe in a porous medium 


\section{Chapter 3}

\section{DESCRIPTION AND USE OF}

\section{THE TOUGH CODE}

Several computer codes are available for modeling strongly heat-driven flow for unsaturated media. Some of them are TOUGH [Pruess, 1987], NORIA [Bixler, 1985] and PETROS [Hadley, 1985]. The TOUGH code is used for this heat pipe study in the repository. TOUGH uses fewer equations and gives better results for two phase flow than NORIA or PETROS. The heat jipe phenomenon and the relevant parameters were studied numerically using the TOUGH code [Pruess. 198i]. TOUGH is a multi-dimensional numerical model for simulating the coupled transport of water. vapor, air and heat in porous and fractured media. It is a member of the MULKOM family of multi-phase, multi-component codes. which is being developed at Lawrence Berkeley Laboratory primarily for geothermal reservoir applications (Pruess. 1983]. The acronym "TOUGH" stands for "Transport Of Unsaturated Groundwater and Heat". It can also be applied to studies of high level nuclear waste emplaced in partially saturated geological media as well as a wide range of problems 
in heat and moisture transfer and in the drying of porous materials.

The deveiopment of the TOUGH code was motivated by problems involving "strongly" heat-driven flow. As temperatures approach or exceed the boiling point of water. vaporization will take place with associated increases in vapor partial pressure and strong forced convection of gas phase. To describe these phenomena. it is necessary to employ a multiphase approach to fluid and heat flow which fully accounts for the movement of liquid and gaseous phases. their transport of latent and sensible heat and phase transitions between liquid and vapor. The gas phase will generally consists of a mixture of water vapor and air. and both these components must be kept track of separately. The TOUGH simulator takes account of the following physical processes. Fluid flow in both liquid and gaseous phases occurs under pressure. viscous. and gravity forces according to Darcy's law with interaction between the phases represented by means of relative permeability functions. Binary diffusion in the gas phase is also considered. Capillary and phase adsorption effects are taken into account for the liquid phase. but no allowance is made for vapor pressure lowering, which will become significant for very strong suction pressures. Also, no allowance is made for hysteresis in either capillary pressure or relative permeability. Air is treated as an ideal gas. and additivity of partial pressures is assumed for air-vapor mixtures.

TOUGH solves three non-linear partial differential equations simultaneously. These consist of the mass conservation equations for water and air, and the energy conservation equation for heat. water, and energy. Air and water can be transported in either the liquid phase. the gas phase. or both. The dissolution of air in water is represented by Henry's law. Heat transport occurs by means of conduction. with thermal conductivity dependent on water saturation. convection, and binary diffusion. 
The code can simulate flow in one. two. or three dimensions because the method of solution is based on a general integrated finite-difference method [Pruess. 1987]. Time stepping is accomplished by a fully implicit procedure.

\subsection{GOVERNING FLOW EQUATIONS}

The goverhing flow equations used in TOUGH are similar to those used in modeling geothermal reservoirs[Pruess, 1985]. The governing mass conservation equation of water can be written as:

$$
\frac{\partial}{\partial \hat{\imath}} \int_{V_{n}} M^{w} d v=\int_{\Gamma_{n}} \overrightarrow{F^{w}} \cdot \hat{n} d \Gamma+\int_{V_{n}} q^{w} d v
$$

where $M^{w}$ :s the mass of water per unit volume in the integrated finite-difference grid block, $V_{n}$ is the volume of the grid block. $\vec{F}^{w}$ is the mass flux of water out the grid block, $\Gamma_{n}$ is the surface area of a grid block, $\hat{n}$ is an outward pointing unit normal vector, and $q^{w}$ is the mass production of water per unit volume. The water is stored in both liquid and gas phases in the above equation. The mass of water per unit volume is, therefore.

$$
M^{w}=\varphi\left(S_{l} \rho_{l} X_{l}^{w}+S_{g} \rho_{g} X_{g}^{w}\right)
$$

where $\phi$ is the matrix porosity, $S_{l}$ is the liquid saturation, $\rho_{l}$ is the liquid density, $X_{l}^{w}$ is the mass fraction of the water in the liquid. $S_{g}$ is the gas saturation $\left(1-S_{l}\right), \rho_{g}$ is the gas density, and $X_{3}^{w}$ is the mass fraction of water in the gas.

A set of equations, similar to the ones for water, can be written for air:

$$
\frac{\partial}{\partial t} \int_{V_{n}} M^{a} d v=\int_{\Gamma_{n}} \vec{F}^{a} \cdot \hat{n} d \Gamma+\int_{V_{n}} q^{a} d v
$$


and

$$
M^{a}=\Phi\left(S_{l} \rho_{l} X_{l}^{a}+S_{3} \rho_{3} X_{3}^{a}\right)
$$

where $M^{a}$ is the mass of air per unit volume in a grid block, $\vec{F}^{a}$ is the mass flux of air out the grid block. $q^{a}$ is the mass production of air per unit volume. $X_{l}^{a}$ is the mass fraction of air in the liquid and $X_{z}^{2}$ is the mass fraction of air in the gas.

The mass flux for the air or water component is simply the sum of the mass fluxes of a comporent over both phases. For water the mass flux is:

$$
\overrightarrow{F^{w}}=\overrightarrow{F_{l}^{w}}+\overrightarrow{F_{g}^{w}}
$$

and for air:

$$
\overrightarrow{F^{a}}=\overrightarrow{F_{l}^{a}}+\overrightarrow{F_{g}^{a}}
$$

where $\vec{F}_{l}^{w}$ is the mass flux of water in the liquid phase, $\vec{F}^{w}$ is the mass flux of water in the gas phase. $\vec{F}_{l}^{a}$ is the mass flux of air in the liquid phase. and $\vec{F}_{g}^{a}$ is the mass flux of air in the gas phase. The mass flux of each component in a phase is governed by Darcy's law and gaseous diffusion. The four equations are:

$$
\begin{gathered}
\overrightarrow{F_{l}^{w}}=-k \frac{k_{r l}}{\mu_{l}} \rho_{l} X_{l}^{w}\left(\nabla P_{l}-\rho_{l} \vec{g}\right) \\
\overrightarrow{F_{l}^{a}}=-k \frac{k_{r l}}{\mu_{l}} \rho_{l} X_{l}^{a}\left(\nabla P_{l}-\rho_{l} \vec{g}\right) \\
\overrightarrow{F_{g}^{w}}=-k \frac{k_{r q}}{\mu_{g}} \rho_{g} X_{j}^{w}\left(\nabla P_{g}-\rho_{g} \vec{g}\right)-D_{v a} \rho_{g} \nabla X_{g}^{w}
\end{gathered}
$$


and

$$
\overrightarrow{F_{3}}=-k \frac{k_{r g}}{\mu_{g}} \rho_{g} X_{\eta}^{a}\left(\nabla P_{g}-\rho_{g} \bar{g}\right)-D_{v a} \rho_{g} \nabla X_{g}^{a}
$$

where $\mathrm{k}$ is the porous medium permeability in the direction of $\hat{n}, k_{r l}$ is the liquid relative permeability, $k_{r g}$ is the gas relative permeability, $\mu_{1}$ is the liquid dynamic viscosity, $\mu_{g}$ is the gas dynamic viscosity, $P_{l}$ is the liquid pressure, $P_{3}$ is the gas pressure, $\vec{g}$ is the acceleration of gravity, and $D_{v a}$ is the binary diffusion coefficient for air-water vapor mixtures. TOUGH cannot handle the off-diagonal terms of the permeability tensor. Furthermore, the code allows only functions to determine the relationship between relative permeability and saturation. and contains a library of functions for these in one of its subroutines. The solubility of air in water is governed by Henry's law:

$$
X_{l}^{a}=\frac{P_{a}}{K_{h}} \frac{M W_{3}}{M W_{w}}
$$

where $P_{a}$ is the air partial pressure, $K_{h}$ is Henry's constant, $M W_{a}$ is the molecular weight of air. and $M W_{w}$ is the molecular weight of water. The binary diffusion coefficient is dependent on both temperature and pressure and is written:

$$
D_{v a}=\tau \phi S_{3} \frac{D_{v a}^{\circ}}{P_{3}}\left[\frac{T-273.15}{273.15}\right]^{\eta}
$$

where $\tau$ is tortuosity, $\mathrm{I}$ is the temperature, and $\nu_{v a}^{\circ}$ is the diffusion coefficient at standard conditions. The parameters $D_{v a}^{\circ}$ and $\eta$ have values $2.13 \times 10^{-5} \mathrm{~m}^{2} / \mathrm{s}$ and 1.80 respectively. Partial pressures are assumed to be additive to determine the gas pressure:

$$
P_{3}=P_{a}+P_{v}
$$


where $P_{z}$ is the air partial pressure and $P_{n}$ is the vapor partial pressure.

The energy conservation equation can be written in an integral form similar to the air and water mass conservation equations. The energy equation is:

$$
\frac{\partial}{\partial t} \int_{V_{n}} I^{h} d v=\int_{\Gamma_{n}} \overrightarrow{F^{h}} \cdot \dot{n} d \Gamma+\int_{V_{n}} q^{h} d v
$$

where $M^{h}$ is the total thermal energy per unit volume in an integrated finite difference grid block, $\vec{F}^{h}$ is the heat flux out of the grid block. and $q^{h}$ is the amount of heat produced per unit volume of grid block. The heat term The total thermal energy term contains contributions from both the rock. liquid. and vapor:

$$
M^{h}=(1-\phi) \rho_{r} c_{r} T+\phi\left(S_{l} \rho_{l} u_{l}+S_{g} \rho_{g} u_{g}\right)
$$

where $\rho_{r}$ is the rock grain density, $c_{r}$ is the rock grain specific heat $u_{l}$ is the specific internal energy of the liquid. and $u_{3}$ is the specific internal thermal energy of the vapor. The heat flux term consists of conductive and convective parts:

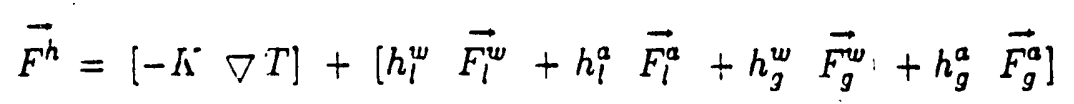

where $K$ is the thermal conductivity of the rock-fluid mixture, $h_{l}^{w}$ is the specific enthalpy of water in the liquid phase. $h_{l}^{a}$ is the specific enthalpy of air in the liquid phase, $h_{g}^{w}$ is the specific enthalpy of water in the gas phase. and $h_{g}^{a}$ is the specific enthalpy of air in the gas phase. The thermal conductivity is allowed to vary with liquid saturation as a linear 
function or a power function by the following equations.

$$
\Pi=K_{d r}+\sqrt{S_{l}}\left(K_{i v e}-K_{d r}\right)
$$

and

$$
K^{*}=K_{d r}+S_{l}\left(K_{w e}-K_{d r}\right)
$$

where $K_{d r}$ is the thermal conductivity of the totally dry porous medium and $K_{w e}$ is the thermal conductivity of the fully saturated porous medium.

The above equations are recast into an integrated finite-difference form for solution. The integrated finite-difference method employed in TOUGH is general. It can be used to solve problems in one, two or three dimension Cartesian or axisymmetric geometries. Because of these general methods. the TOUGH code requires volumes for the grid blocks, distances between the grid blocks. and interfacial areas between the grid blocks as input data. Because of its generality, TOUGH is not limited to rectangular or rectangular-parallelopiped grid blocks. The grid blocks can be any shape such as pentagons or tetrahedrons, but the aspect ratio must not be extreme.

TOUGH handles several types of boundary conditions. If a grid block edge is not connected to another grid block. then that edge becomes a zero flux boundary condition for all the dependent variables. If a grid block is assigned an extremely large volume relative to other grid blocks. then the dependent variables are essentially fixed with respect to time. Time varying flux boundary conditions can be implemented by using the time-varying source/sink capability in TOUGII. 


\section{Chapter 4}

\section{DESCRIPTION OF MODELING}

As stated earlier. the host thck is modeled as a partially saturated porous medium with expiicit fractures. An infinite linear string of waste packages, which is intersected by a set of plane. parallel fractures at a spacing of $0.22 \mathrm{~m}$ is considered. The waste package is assumed to be of $4.5 \mathrm{~m}$ long. The geometry of the model selected in this study is similar to the one Pruess used earlier [Pruess et al., 1990].

Fig.4(a) shows the geometry of the model used in this study. One dimensional radial geometry is selected. so that the fluid and heat. flow radially inwards or outward. The fracture is assimed to be $0.002 \mathrm{~m}$ wide. Elements close to the fracture are made thinner and the thickness of the elements increases as the distance from the fracture increases in the vertical directions. The total thickness of the fracture layer and three layers of the matrix will be equal to $0.11 \mathrm{~m}$ because of symmetry. The boundaries are assumed to be a $t$ a radius of 20 meters from the center of the cannister. Since most of the hydrologic and thermal phenomena occur in the near field around the cannister. elements close to the cannister are made thinner in the radial direction also. As the radial distance increases. the element 
thickness also increases.

Modeling of the fractured porous medium consists of two parts. fracture modeling and matrix modeling. In this modeling, all of the fracture properties are tested for a renge of values corresponding to the various fractured tuff units found in Yucca Mountain Peters et al.. 1984]. In particular. parameters used in this heat pipe study are from samples G4-1F. G4-2F, G4-3F, G4-4F, and G4-5F.

Matrix properties are kept constant during the fracture modeling. In modeling the matrix, all the matrix properties are tested for the different values of the fractured tuffs found in Yucca Mountain. In this case. the fracture properties are kept constant.

\subsection{FRACTURE MODELING}

The fracture properties tested were permeability, thermal conductivity, tortuosity, porosity, and specific heat. When each of the above properties is tested, the remaining four parameters are kept constant. In this way, the exact influence of each property is well understood. The initial conditions are given by $T=26^{\circ} \mathrm{C}$ and $P=10^{5} P_{a}$. A liquid saturation of 0.80 is taken for the matrix. The suction pressure in the fracture and the matrix is assumed to be equal for the fracture-matrix equilibrium. The suction pressure is a function of the liquid saturation. The liquid saturation in the fracture is determined from a known matrix liquid saturation and the equality of the suction pressures in the fracture and the matrix. A matrix liquid saturation of 0.80 produces a fracture liquid saturation of 0.0099 . An irreducible liquid saturation of 0.01 is assumed for the fracture so that the fracture liquid saturation is beln: the irreducible Liquid saturation and this makes the flow immobile. 


\subsubsection{RELATIVE PERMEABILITY \& CAPILLARY PRESSURE}

Several functions are available for the relative permeability and the capillary pressure that can be used for the modeling of fracture. Linear functions are selected for the relative permeability and capillary pressure in the fracture for the modeling of the fracture in all the simulation:

The capillary pressure in the fracture is described by the linear function. For the linear function. the different cases are given as follows.

$$
\begin{gathered}
P_{\text {cap }}=0 \quad \text { for } S_{l, f} \geq S_{l s . f} \\
P_{\text {cap }}=-P_{\text {max }} \frac{S_{l s . f}-S_{l, f}}{S_{l s, f}-S_{l r, f}} \quad \text { for } S_{l r, f}<S_{l, f}<S_{l s, f} \\
P_{\text {cap }}=-P_{\text {max }} \quad \text { for } S_{l, f} \leq S_{l r . f} \quad
\end{gathered}
$$

where $S_{l, f}, S_{l r, f}, S_{l s . f}$, and $P_{\max }$ represent the liquid saturation. the irreducible liquid saturation. the scaled liquid saturation. and the maximum capillary pressure in the fracture respectively. The only restriction for the linear function in the above relations is that $S_{l s, f}>S_{l r, f}$

Different parametric values associated with the linear capillary pressure function used for the fracture modeling are shown in table 4.1 below. 
Table 4.1

\begin{tabular}{|l|l|}
\hline Parameter & Numerical value \\
\hline$P_{\text {max }}$ & $5 \times 10^{8} P_{a}$ \\
\hline$S_{l \text { r.f }}$ & 0.01 \\
\hline$S_{l . f}$ & 0.0099 \\
\hline
\end{tabular}

The relative permeabilities are also described by a linear function. Relative permeabilities for the different liquid saturations are given as follows.

$$
\begin{gathered}
k_{r l . f}=\frac{S_{l . f}-S_{l r . f}}{S_{l s . f}-S_{l r . f}} \quad \text { for } S_{l r, f} \leq S_{l, f} \leq S_{l s . f} \\
k_{r l, f}=0 \quad \text { for } S_{l, f} \leq S_{l r, f} \\
k_{r l, f}=1 \quad \text { for } S_{l, f} \geq S_{l s . f}
\end{gathered}
$$

Similariy, the relative permeability of the gas in the fracture is obtained from the following relations.

$$
\begin{gathered}
k_{r g, f}=\frac{S_{g, f}-S_{g r, f}}{S_{g s, f}-S_{g r, f}} \\
k_{r g, f}=0 \quad \text { for } S_{g, f} \leq S_{g r, f} \\
k_{r g, f}=1 \quad \text { for } S_{g, f} \geq S_{g s, f}
\end{gathered}
$$

where $S_{g, f}, S_{g r, j}, S_{g s, j}, k_{r l, f}$. and $k_{r g, j}$ represent the gas saturation. the irreducible gas saturation. the scaled gas saturation. the relative permeability of the liquid, and the relative permeability of the gas in the fracture respectively. 
Different parametric values associated with the linear relative permeability function used or the fracture modeling are shown in table 4.2 below.

Table 4.2

\begin{tabular}{|l|l|}
\hline Parameter & Numerical value \\
\hline$S_{l . f}$ & 0.0099 \\
\hline$S_{\text {lr.f }}$ & 0.01 \\
\hline$S_{l s . f}$ & 1 \\
\hline
\end{tabular}

Initially, the majority of the fractures will be drained and a small amount of liquid may be present on the rough surfaces of the fracture due to adsorption. The thin film of liquid present on the walls of the drained fractures has a low mobility [Pruess et al., 1990]. This is due to the intermolecular forces. Data on the characteristic curves of the relative permeability and the capillary pressure are available only for the matrix and not for the fracture. The relative permeability and the capillary pressure for the fracture are found based upon the matrix liquid saturation and the equality of the fracture and the matrix capillary pressures. An immobile liquid saturation $S_{l r . f}$ can be assumed such that the relative permeability of the liquid in the fracture $k_{r l, f}=0$ for $S_{l, f} \leq S_{l r, f}$ and this is the fully immobile case. In the fully mobile case, the relative permeability is unity so that

$$
k_{r l, f}=1 \quad \text { for } S_{l s, f} \leq S_{l, f} \leq 1
$$

The partly mobile case occurs when $S_{l, f} \leq S_{l, f} \leq S_{l s, f}$ and $k_{r l, f}$ in this case varies linearly irom 0 to 1 . For the linear function, relative permeability of the gas in the fracture $k_{r g, f}$ 
increases lineariy from 0 to 1 in the range $S_{g r, j} \leq S_{3} \leq S_{g g, f}$

Many simulations were done using the TOUGH code which invoived the testing of properties such as permeability, porosity, thermal conductivity, tortuosity, and specific heat for the fracture. Each of the above properties are tested for a range of values and the resulting effects on the heat pipe region are examined. The tested variables and their standard values for comparison are given in table 4.3 below.

Table 4.3

\begin{tabular}{|l|l|l|}
\hline Testing variable & Reference values & Range \\
\hline Permeability & $1.1 \times 10^{-11} \mathrm{~m}^{2}$ & $2.7 \times 10^{-7}-1.1 \times 10^{-11} \mathrm{~m}^{2}$ \\
\hline Thermal conductivity & $1.6 \mathrm{~W} / \mathrm{m}^{\circ} \mathrm{C}$ & $1.16-2.25 \mathrm{~W} / \mathrm{m}^{\circ} \mathrm{C}$ \\
\hline Tortuosity & 0.25 & $0-0.35$ \\
\hline Porosity & 0.90 & $0.20-0.90$ \\
\hline Specific heat & $768 \mathrm{~J} / \mathrm{kg}^{\circ} \mathrm{C}$ & $400-1250 \mathrm{~J} / \mathrm{kg}^{\circ} \mathrm{C}$ \\
\hline
\end{tabular}

\subsubsection{FRACTURE PERMEABILITY}

The maximum permeability used for the simulation was the parallel plate permeability. This value is taken as $k_{f}=2.7 \times 10^{-i} \mathrm{~m}^{2}$. The minimum fracture permeability tested was $1.1 \times 10^{-11} \mathrm{~m}^{2}$. This value corresponds to the maximum matrix permeability. Temperature and liquid saturation profiles were plotted at 51 days and at 1 year for all the four values and compared for any significant change in the heat pipe region. From these, it can be concluded that the fracture permeability does not have any significant effect on the heat 
?ipe performance. Distances are always measured from the center line of the cannister. A distance of $0.27 \mathrm{~m}$ in table 4.4 corresponds to the bore hole wall. The temperature distribution ior different fracture permeabilities are given in table 4.4 below. From these. the effect of fracture permeability on the performance of heat pipe is found to be negligibly small.

Table 4.4

\begin{tabular}{|l|l|l|l|l|l|l|l|l|}
\hline \multirow{2}{*}{$\begin{array}{l}\text { Run } \\
\#\end{array}$} & \multirow{2}{*}{\begin{tabular}{l}
$k_{f}$ \\
\cline { 3 - 9 }
\end{tabular}} & \multicolumn{6}{|c|}{ Temperature in ${ }^{\circ} \mathrm{C}$ at certain distances } \\
\cline { 3 - 9 } & $.27 \mathrm{~m}$ & $.61 \mathrm{~m}$ & $.11 \mathrm{~m}$ & $.84 \mathrm{~m}$ & $.97 \mathrm{~m}$ & $1.13 \mathrm{~m}$ & $1.52 \mathrm{~m}$ \\
\hline 1 & $2.7 \times 10^{-i}$ & 149.0 & 100.5 & 100.4 & 100.1 & 99.47 & 92.74 & 75.64 \\
\hline 2 & $1.1 \times 10^{-9}$ & 148.9 & 100.4 & 100.3 & 100.1 & 99.45 & 92.67 & 75.65 \\
\hline 3 & $1.1 \times 10^{-10}$ & 148.9 & 100.4 & 100.2 & 100.1 & 99.51 & 92.71 & 75.58 \\
\hline 4 & $1.1 \times 10^{-11}$ & 148.5 & 100.5 & 100.3 & 100.2 & 99.58 & 92.53 & 75.61 \\
\hline
\end{tabular}

The heat pipe is affected considerably by the mobility of the liquid in the fracture[Pruess et al.. 1990]. Mobility of the liquid in the fracture is an important issue and has a strong impact on the heat pipe ptrformance.

The liquid in the fracture is made mobile as follows. The capillary pressure in the matrix is found for an initial matrix liquid saturation of 0.80 . By equating the suction pressures in the matrix and the fracture. the fracture liquid saturation is obtained so that the parameters for the linear capillary pressure function in the fracture are adjusted. If the computed fracture liquid saturation $S_{l, f}$ is greater than the irreducible fracture liquid 
saturation $S_{l r . f}$, the mobile case is achieved. A fracture liquid saturation of 0.016 is obtained for values of $S_{l s . f}=0.5$ and $S_{l r . f}=0.0099$. Since $S_{l, f}$ is greater than $S_{l r . f}$, this represents the mobile case. Simulations are carried out using the new fracture iqquid saturation.

The same fracture permeabilities were used for both mobile and immobile cases. The temperature distribution for different permeabilities for the mobile case is shown in table 4.5 . $A$ large difference in temperatures at the bore hole wail $(0.27 \mathrm{~m})$ can be found between the mobile and immobile cases. The wall temperature for the immobile case was approximately $149^{\circ} \mathrm{C}$. The length of the heat pipe was $0.36 \mathrm{~m}$ in this case. No liquid saturation was obtained in the near field. The heat pipe effect was absent at or near the bore hole wall.

The heat pipe periormance was totally different for the mobile case compared to the immobile case. For the mobile case. the maximum temperature remains near $100{ }^{\circ} \mathrm{C}$ for all the permeabilities and liquid saturation is obtained even at the emplacement hole near the heat source. The near field is always in the two phase conditions without any dry out condition. Thus the heat pipe region was extended up to the bore hole wall and it is approximately $1.15 \mathrm{~m}$ in length. Mobility of the liquid in the fracture brings the moisture into the bore hole surface. Again this shows no eflect of permeability. 
Table 4.5

\begin{tabular}{|l|l|l|l|l|l|l|l|l|}
\hline \multirow{2}{*}{$\begin{array}{l}\text { Run } \\
\text { \# }\end{array}$} & $k_{f}$ & \multicolumn{6}{|c|}{ Temperature in ${ }^{\circ} \mathrm{C}$ at certain distances } \\
\cline { 3 - 9 } & $.27 \mathrm{~m}$ & $.61 \mathrm{~m}$ & $.71 \mathrm{~m}$ & $.84 \mathrm{~m}$ & $.97 \mathrm{~m}$ & $1.13 \mathrm{~m}$ & $1.52 \mathrm{~m}$ \\
\hline 1 & $2.7 \times 10^{-i}$ & 102.0 & 100.5 & 100.2 & 100.0 & 99.10 & 97.94 & 83.45 \\
\hline 2 & $1.1 \times 10^{-9}$ & 102.1 & 100.8 & 100.5 & 100.2 & 99.32 & 98.70 & 84.01 \\
\hline 3 & $1.1 \times 10^{-10}$ & 102.2 & 100.9 & 100.7 & 100.4 & 99.78 & 98.83 & 84.21 \\
\hline 4 & $1.1 \times 10^{-11}$ & 102.5 & 101.1 & 100.8 & 100.5 & 99.95 & 98.91 & 84.77 \\
\hline
\end{tabular}

Simulations were also carried out for a different relative permeability function for the immobile case. Grant's curve is a fourth order power curve and is the next available higher order function to the linear function. This relative permeability function can be described through the following equations.

$$
\begin{gathered}
S^{*}=\frac{\left(S_{l, f}-S_{l r, f}\right)}{1-\left(S_{l r, f}+S_{l s, f}\right)} \\
k_{r l, f}=\left(S^{*}\right)^{4} \\
k_{r g, f}=1-k_{r l, f}
\end{gathered}
$$

The only restriction is given by the following inequality.

$$
S_{i r, f}+S_{l s, f}<1
$$

$\dot{s}_{i r f}=0.01$ and $S_{l s . f}=0.15$ are selected so that the above restriction is fulfilled for the immobile case. 
The results of the simulations for the immobile case using Grant's curves are given in rable 4.6 below. Temperature distribution in the near field for the Grant's curves showed a slight increase in temperature compared to the linear functions. The nature and the extent . of the heat pipe remain unaffected by this change in fracture relative permeability function.

Table 4.6

\begin{tabular}{|l|l|l|l|l|l|l|l|l|}
\hline \multirow{2}{*}{$\begin{array}{l}\text { Run } \\
\#\end{array}$} & \multirow{2}{*}{$\begin{array}{l}k_{f} \\
\left(\mathrm{~m}^{2}\right)\end{array}$} & \multicolumn{6}{|c|}{ Temperature in ${ }^{\circ} \mathrm{C}$ at certain distances } \\
\cline { 3 - 9 } & $.27 \mathrm{~m}$ & $.61 \mathrm{~m}$ & $.71 \mathrm{~m}$ & $.84 \mathrm{~m}$ & $.97 \mathrm{~m}$ & $1.13 \mathrm{~m}$ & $1.52 \mathrm{~m}$ \\
\hline 1 & $2.7 \times 10^{-i}$ & 148.6 & 100.8 & 100.6 & 100.3 & 100.1 & 93.30 & 76.77 \\
\hline 2 & $1.1 \times 10^{-9}$ & 148.7 & 100.8 & 100.9 & 100.5 & 100.3 & 93.88 & 76.98 \\
\hline 3 & $1.1 \times 10^{-10}$ & 149.0 & 100.9 & 101.0 & 100.7 & 100.4 & 93.97 & 77.02 \\
\hline 4 & $1.1 \times 10^{-11}$ & 149.3 & 101.1 & 101.3 & 101.0 & 99.96 & 92.22 & 86.72 \\
\hline
\end{tabular}

Linear functions were used for the capillary pressure in all the previous simulations. The effect of the fracture capillary pressure function on the temperature and the heat pipe region were examined by selecting a different capillary pressure function and comparing the temperatures and the extent of the heat pipe. Capillary pressures for the Sandia function in the fracture for the different values of $S_{l . f}$ are obtained as followsiHayden et al., 1983; Genuchten, V., 1980!.

$$
\begin{gathered}
P_{c a p}=0 \quad \text { for } S_{l, f} \geq S_{l \text { s.f }} \\
P_{\text {cap }}=-P_{0}\left[\left(S^{*}\right)^{\frac{-1}{\lambda}}-1\right]^{1-\lambda} \quad \text { for } S_{l . f}>S_{l \text { r.f }}
\end{gathered}
$$




$$
\begin{gathered}
S^{*}=\frac{S_{l . f}-S_{l . f}}{S_{l . f}-S_{l r . f}} \\
P_{\text {rap }}=-P_{\text {max }} \quad \text { for } P_{0}\left[\left(S^{*}\right)^{\frac{-1}{\lambda}}-1\right]^{1-\lambda} \geq P_{\text {max }}
\end{gathered}
$$

where $\lambda$ is a parameter given by $\lambda=1-\frac{1}{3}, P_{0}$ is the minimum capillary pressure in the fracture and $\beta$ is a curve-fit parameter for the Van Genuchten curve and it is taken as 1.85 [Peters et al., 1984].

The mobile case simulations were done using the Sandia function. The same fracture permeabilities were used for both the Sandia function and the linear function. These simulations indicated no significant change in the temperatures. The temperatures at different points using the Sandia function were exactly identical to the values obtained for the linear function. The nature and the extent of the heat pipe region were also the same for both the functions. From these results. the heat pipe performance is not found to be sensitive to the capillary pressure function.

\subsubsection{FRACTURE THERMAL CONDUCTIVITY}

Since the fracture mainly consists of air. the fracture thermal conductivity is approximated as the thermal conductivity of air. Simulations were done for $0.03, .25, .75$, and 1.6 $\mathrm{W} / \mathrm{m}^{\circ} \mathrm{C}$. The minimum value of $0.03 \mathrm{~W} / \mathrm{m}^{\circ} \mathrm{C}$ corresponds to the thermal conductivity of air and the maximum value of $1.6 \mathrm{~W} / \mathrm{m}^{\circ} \mathrm{C}$ corresponds to the average thermal conductivity of the rock. The two intermediate values were arbitrarily chosen. The temperatures at certain distances away from the heat source for different thermal conductivities are shown in table $4 . \bar{i}$. 
Table 4.7

\begin{tabular}{|l|l|l|l|l|l|l|l|l|l|}
\hline \multirow{2}{*}{ Run } & $\mathrm{K}$ & \multicolumn{7}{|c|}{ Temperature in ${ }^{\circ} \mathrm{C}$ at certain distances } \\
\cline { 3 - 10 }$\#$ & $\left(W / \mathrm{m}^{\circ} \mathrm{C}\right)$ & $.27 \mathrm{~m}$ & $.61 \mathrm{~m}$ & $.71 \mathrm{~m}$ & $0.84 \mathrm{~m}$ & $0.97 \mathrm{~m}$ & $1.13 \mathrm{~m}$ & $1.52 \mathrm{~m}$ & $5.52 \mathrm{~m}$ \\
\hline 1 & 1.60 & 147.1 & 100.8 & 100.5 & 100.2 & 99.9 & 90.1 & 73.2 & 27.8 \\
\hline 2 & 0.75 & 147.3 & 100.9 & 100.5 & 100.0 & 99.8 & 90.0 & 72.8 & 27.8 \\
\hline 3 & 0.25 & 147.0 & 100.5 & 100.2 & 100.0 & 99.7 & 90.3 & 73.1 & 27.9 \\
\hline 4 & 0.03 & 147.3 & 100.7 & 100.3 & 100.1 & 99.9 & 90.2 & 73.5 & 28.1 \\
\hline
\end{tabular}

From this table, it can be seen that the heat pipe region and the condensation point remain unaffected by the changes in the thermal conductivity.

\subsubsection{FRACTURE TORTUOSITY}

Tortuosity accounts for the effects of binary diffusion. Binary diffusion means the diffusion of water vapor in air. Binary diffusion coefficient is dependent on both temperature and pressure. Tortuosity is dependent on pore geometry. Tortuosity factor and binary diffusion coefficient are related through the following equation /Vargaftik. 1975: Walker et al. 1981].

$$
D_{v a}=\tau \oplus S_{g} \frac{D_{* a}^{\circ}}{P_{g}}\left[\frac{T+273.15}{273.15}\right]^{\theta}
$$

where $D_{v a}$ is the binary diffusion coefficient corrected for the temperature and the pressure, - is the tortuosity factor, $P_{3}$ and $S_{3}$ are the pressure correction. T is the temperature, $\phi$ is the porosity, and $D_{v a}^{\circ}$ is the diffusivity of water vapor in air at standard conditions. A pressure of one bar and a temperature of $0^{\circ} \mathrm{C}$. are taken as the standard conditions. $D_{v a}^{\circ}$ and $\theta$ are parameters which have values of $2.13 \times 10^{-5} \mathrm{~m}^{2} / \mathrm{s}$ and 1.30 . respectively, for a 
pressure oi one bar and a temperature of $0^{\circ} \mathrm{C}$. The terms $T$ and 0 represent the effect of porous media properties on the binary diffusion.

Tortuosity factor $\boldsymbol{T}$ is defined as a function of the ratio between the shortest linear distance in the direction of flow to the length of tortuous fluid paths through the pores. For a porous media. a reference value of 0.4 is taken as the tortuosity factorịear et al.. 1968]. Based on this reference value, different tortuosities are selected for the fracture. The effect of tortuosity on the heat pipe performance is studied in detail from the results of four simulations made with tortuosities of $0.50,0.40,0.25$ and 0.15 . The temperature and the liquid saturation at selected distances remained constant for these simulations. The heat pipe region was the same for the above cases. Temperatures corresponding to different tortuosities after $j 1$ davs are shown in table 4.8 below.

Table 4.8

\begin{tabular}{|l|l|c|c|c|c|c|c|c|}
\hline \multirow{2}{*}{$\begin{array}{l}\text { Run } \\
\#\end{array}$} & \multirow{2}{*}{$\begin{array}{l}\text { Variable } \\
\text { (Tortuosity) }\end{array}$} & \multicolumn{6}{|c|}{ Temperature in ${ }^{\circ} \mathrm{C}$ at certain distances } \\
\cline { 3 - 9 } & $.27 \mathrm{~m}$ & $.61 \mathrm{~m}$ & $.71 \mathrm{~m}$ & $.84 \mathrm{~m}$ & $.97 \mathrm{~m}$ & $1.13 \mathrm{~m}$ & $1.52 \mathrm{~m}$ \\
\hline 1 & 0.50 & 149.0 & 100.8 & 100.4 & 100.1 & 99.9 & 90.3 & 73.8 \\
\hline 2 & 0.40 & 148.9 & 100.9 & 100.4 & 100.2 & 99.8 & 90.5 & 74.0 \\
\hline 3 & 0.25 & 148.9 & 100.8 & 100.5 & 100.3 & 99.8 & 90.5 & 74.0 \\
\hline 4 & 0.15 & 149.0 & 100.7 & 100.5 & 100.2 & 99.9 & 90.6 & 73.9 \\
\hline
\end{tabular}

From the above table. it is clearly shown that the tortuosity does not have any effect on the heat pipe behavior. 


\subsubsection{FRACTURE POROSITY}

The fracture is assumed to be highly porous. The fracture porosity was tested for a naximum value of 0.90 and three other values $0.8,0.7$. and 0.6 . These values are taken from iPeters et al.. 1984!. Temperatures corresponding to the different porosities at 51 days are given in table 4.9 . These results show no significance of porosity on the performance of the heat pipe.

Table 4.9

\begin{tabular}{|l|l|l|l|l|l|l|l|l|l|}
\hline \multirow{2}{*}{$\begin{array}{l}\text { Run } \\
\#\end{array}$} & \multirow{2}{*}{$\begin{array}{l}\text { Variable } \\
(\mathrm{Py})\end{array}$} & \multicolumn{7}{|c|}{ Temperature in ${ }^{\circ} \mathrm{C}$ at certain distances } \\
\cline { 2 - 10 } & $.27 \mathrm{~m}$ & $.71 \mathrm{~m}$ & $0.84 \mathrm{~m}$ & $0.97 \mathrm{~m}$ & $1.13 \mathrm{~m}$ & $1.52 \mathrm{~m}$ & $5.52 \mathrm{~m}$ \\
\hline 1 & 0.90 & 149.0 & 100.8 & 100.4 & 100.1 & 99.8 & 90.4 & 73.8 & 28.3 \\
\hline 2 & 0.80 & 148.8 & 100.9 & 100.3 & 100.2 & 99.8 & 90.3 & 74.0 & 28.2 \\
\hline 3 & 0.70 & 148.7 & 100.7 & 100.3 & 100.1 & 99.7 & 90.3 & 74.2 & 28.2 \\
\hline 4 & 0.60 & 149.7 & 100.6 & 100.4 & 100.2 & 99.9 & 90.2 & 74.0 & 28.1 \\
\hline
\end{tabular}

\subsubsection{FRACTURE SPECIFIC HEAT}

The specific heat of the fracture selected was based on the matrix specific heat. The average heat capacities were given as $i 68 \mathrm{~J} / \mathrm{kg}{ }^{\circ} \mathrm{C}$ for both fracture and matrix. The other fracture $c_{p}$ values chosen for the heat pipe study were $450 \mathrm{~J} / \mathrm{kg}^{\circ} \mathrm{C}$ and $1250 \mathrm{~J} / \mathrm{kg}^{\circ} \mathrm{C}$ Peters et al., 1984]. Since the fracture mostly consists of air. the specific heat of air $\left(c_{p}=\right.$ $\left.1005 \mathrm{~J} / \mathrm{kg}{ }^{\mathrm{J}} \mathrm{C}\right)$ is used in one simulation. The results of these simulations showed no significance on the performance of heat pipe. Temperatures corresponding to the different heat rapacities at 51 days are given in table +.10 . 
Table 4.10

\begin{tabular}{|l|l|l|l|l|l|l|l|l|l|}
\hline \multirow{2}{*}{ Run } & $C_{p}$ & \multicolumn{6}{|c|}{ Temperature in ${ }^{\circ} C$ at certain distances } \\
\cline { 2 - 9 }$\#$ & $\left(\mathrm{~J} / \mathrm{kg}{ }^{\circ} \mathrm{C}\right)$ & $.27 \mathrm{~m}$ & $.61 \mathrm{~m}$ & $.71 \mathrm{ru}$ & $.84 \mathrm{~m}$ & $.97 \mathrm{~m}$ & $1.13 \mathrm{~m}$ & $1.52 \mathrm{~m}$ & $5.52 \mathrm{~m}$ \\
\hline 1 & 1250 & 149.0 & 100.8 & 100.4 & 100.1 & 99.9 & 90.3 & 73.8 & 28.1 \\
\hline 2 & 1005 & 149.0 & 100.7 & 100.5 & 100.2 & 99.9 & 90.6 & 73.9 & 28.0 \\
\hline 3 & $i 68$ & 148.5 & 100.8 & 100.5 & 170.2 & 99.9 & 90.2 & 74.5 & 28.4 \\
\hline 4 & 450 & 148.6 & 100.6 & 100.4 & 100.3 & 99.7 & 90.1 & 74.6 & 28.1 \\
\hline
\end{tabular}

\subsection{FRACTURE MODELING RESULTS}

The iracture properties such as permeability. thermal conductivity, porosity, tortuosity, and specific heat do not change the heat pipe region and the condensation point. The most important factor in the fracture modeling was the mobility of the liquid in the fracture. Mobility of the liquid in the fracture changes the heat pipe phenomenon to a great extent compared to the immobile case. For the immobile case. the heat pipe region was found to be smail. The temperature at the bore hole wall was approximately $149^{\circ} \mathrm{C}$ without any two phase conditions at the wall. For the mobile case. the heat pipe region was extended Lp to the bore hole wall with a stronger heat pipe region. This was unaffected by the perneability value. Two phase conditions always prevailed at the bore hole wall. The near field temperature was always near $100^{\circ} \mathrm{C}$ without any àry out condition. The fracture was modeled using different relative permeability and capillary pressure functions for comparing the resuitu. The relative permeability and the capillary pressure functions did not change the heat pipe significantly. 


\subsection{MATRIX MODELING}

The matrix is modeled by keeping the fracture properties constant at : 'eir reference values. The initial liquid saturation in the matrix is taken as 0.80 . Other initial conditions are given by $T=26{ }^{\circ} \mathrm{C}$ and $P=10^{5} P_{2}$. The tested variables and their standard values for comparison are shown in table 4.11 below. The matrix is tested for each property by keeping the remaining properties fixed as in the case of fracture modeling.

Table 4.11

\begin{tabular}{|l|l|l|}
\hline Testing variable & Reference values & Range \\
\hline Permeability & $3.3 \times 10^{-17} \mathrm{~m}^{2}$ & $3.3 \times 10^{-18}-3.3 \times 10^{-15} \mathrm{~m}^{2}$ \\
\hline Specific heat & $768 \mathrm{~W} / \mathrm{m}^{\circ} \mathrm{C}$ & $400-1250 \mathrm{~W} / \mathrm{m}^{\circ} \mathrm{C}$ \\
\hline Tortuosity & 0.25 & $0-0.35$ \\
\hline Porosity & 0.90 & $0.20-0.90$ \\
\hline Th. conductivity & $1.6 \mathrm{~J} / \mathrm{kg}^{\circ} \mathrm{C}$ & $1.16-2.25 \mathrm{~J} / \mathrm{kg}^{\circ} \mathrm{C}$ \\
\hline
\end{tabular}

\subsubsection{CHARACTERISTIC FUNCTIONS}

For the matrix modeling, Sandia functions [Hayden $a t$ al.. 1983; Van Genuchten, 1980] are used for both relative permeability and capillary pressure. As described earlier, linear functions are used for both relative permeability and sapillary pressure in the fracture. For the matrix modeling, Sandia functions for the capillary pressure can be described as follows.

$$
P_{\text {cap }}=0 \quad \text { for } S_{l, m} \geq S_{l s . m}
$$




$$
\begin{aligned}
& P_{\text {cap }}=-P_{0}\left[\left(S^{*}\right)^{\frac{-1}{\lambda}}-1\right]^{1-\lambda} \text { for } S_{l . m}>S_{l r . m} \\
& P_{c a p}=-P_{\max } \quad \text { for } P_{0}\left[\left(S^{*}\right)^{\frac{-1}{\lambda}}-1\right]^{1-\lambda} \geq P_{\max }
\end{aligned}
$$

Sandia functions for the relative permeability in the matrix for different values of $S_{l, m}$ are obtained as follows.

$$
\begin{gathered}
S^{*}=\frac{S_{l, m}-S_{l r, m}}{S_{l s, m}-S_{l r, m}} \\
k_{r l, m}=\sqrt{S^{*}}\left[1-\left[1-S^{* \frac{1}{\lambda}}\right]^{\lambda}\right]^{2} \quad \text { for } S_{l, m}<S_{l s, m} \\
k_{r i, m}=1 \quad \text { for } S_{l, m} \geq S_{l s . m} \\
k_{r g, m}=1-k_{r l, m}
\end{gathered}
$$

where $S_{l, m}, S_{l, m}, S_{l s, m}, k_{r l, m}, k_{r g, m}$, and $\lambda$ represent the liquid saturation, irreducible liquid saturation. scaled liquid saturation. liquid relative permeability, gas relative permeability, and the Van Genuchten curve fit parameter respectively in the matrix.

The numerical values used in the above equations are given in Table 4.12 below. 
Table 4.12

\begin{tabular}{|l|l|}
\hline Parameter & Numerical value \\
\hline$\lambda$ & 0.45 \\
$S_{l r . m}$ & $9.6 \times 10^{-4}$ \\
$P_{0}$ & $1.39 \times 10^{6} P_{a}$ \\
$P_{\max }$ & $5 \times 10^{8} P_{a}$ \\
$S_{l s . m}$ & 1 \\
$S_{l . m}$ & 0.80 \\
\hline
\end{tabular}

\subsubsection{MATRIX PERMEABILITY}

The effect of matrix permeability for the heat pipe region was tested using the TOUGH code for a range of utatrix permeabilities. These values correspond to the different fractured tuffs found at Yucca Mountain as taken from [Peters et al., 1984]. Resuits were obtained without any convergence problem for matrix permeabilities in the range $3.2 \times 10^{-18}-3.2 \times$ $10^{-15} \mathrm{~m}^{2}$ for the particular geometry selected. Within this range, the simulations gave very good results and simulations were done for an extended period of time. Stability problems were found to occur beyond this range. The mass flux will be very small for very small permeabilities. Large time steps and large volume elements are required for lower matrix permeabilities to avoid convergence problems. The mass flux will be large for very high permeabilities. Small time steps and small elements are required for very high permeabilities to overcome this convergence problem. The different matrix permeabilities tested for the heat pipe phenomenon are shown in table 4.13 . 
Table 4.13

\begin{tabular}{|l|l|}
\hline Run $\#$ & Permeability $\left(\mathrm{m}^{2}\right)$ \\
\hline 1 & $3.2 \times 10^{-18}$ \\
2 & $3.2 \times 10^{-17}$ \\
3 & $3.2 \times 10^{-16}$ \\
4 & $3.2 \times 10^{-15}$ \\
\hline
\end{tabular}

Temperature profiles at 51 days for different matrix permeabilities are plotted as shown in Fig.4(b). Both the fracture and the matrix are in equilibrium. For the graph corresponding to the minimum permeability of of $3.2 \times 10^{-18} \mathrm{~m}^{2}$. the maximum temperature observed at the end of 51 days was $160^{\circ} \mathrm{C}$. The temperature remains fairiy constant near $100^{\circ} \mathrm{C}$ for a distance $0.14 \mathrm{~m}$ from element 8 to element 9 . In this region, a very high liquid saturation is obtained. This region is the heat pipe region. The point corresponding to the maximum liquid saturation is the point of condensation. The heat pipe region is very small for this permeability. For the graph plotted for a permeability of $3.2 \times 10^{-17} \mathrm{~m}^{2}$, the temperature remained constant for a distance $0.37 \mathrm{~m}$ from element 6 to element 9 . The heat pipe region here is much longer than the previous case. For a matrix permeability of $3.2 \times 10^{-16} \mathrm{~m}^{2}$, the temperature remains approximately constant for a considerable distance from the canister(heat source), so the heat pipe region is still longer compared to the previous cases. The maximum temperature is dropped to $102^{\circ} \mathrm{C}$ and some liquid saturation is obtained at the bore hole wall. The heat pipe phenomenon for the maximum permeability of $3.2 \times 10^{-15} \mathrm{~m}^{2}$ is similar to the case of $k_{m}=3.2 \times 10^{-16} \mathrm{~m}^{2}$ without much change in temperature and liquid saturation. 
Fig.4(c) shows the liquid saturation proñle at 51 days for a matrix permeability of $k_{m}=3.2 \times 10^{-18} \mathrm{~m}^{2}$. High liquid saturation is obtained in the heat pipe region and it starts dropping beyond this region. At some distance away from the heat source. the initial Liquid saturation is obtained. Fig.4(d) describes the liquid saturation profile for a matrix permeability of $k_{m}=3.2 \times 10^{-15} \mathrm{~m}^{2}$. In this case, high liquid saturation is obtained even for the elements close to the heat source. so the heat pipe region is extended up to the bore hole wall for the maximum permeability case.

From these graphs. it can be seen that the higher temperature distributions are obtained for low matrix permeabilities. As the the permeability is increased. the flow of liquid towards the heat source is also increased. The heat pipe region is extended up to the bore hole wall so that there is some liquid saturation at the bore hole wall. The point of condensation and the heat pipe region will be shifted closer to the heat source due to the increase in convective heat transfer.

Fig.4(e) compares the the temperature profiles at 1 year for the different permeabilities tested. The temperature profiles at 1 year for the corresponding matrix permeabilities show the same trend. At 1 year. the maximum temperature obtained is $220^{\circ} \mathrm{C}$ for the lowest permeability and it is just $104^{\circ} \mathrm{C}$ for the maximum permeability. It can be seen that the heat pipe region is shifted away from the heat source with the increase in simulation period. As the permeability increases. the temperature attains a steady state more quickly. For the maximum matrix permeability value of $k_{m}=3.23 \times 10^{-15} \mathrm{~m}^{2}$, the steady state temperature is reached after one year after the emplacement of the canister. For the lower permeabilities, the temperature is still increasing at the same point and not reaching a constant temperature. It can be seen that the heat pipe region approaches the heat source 
:vith the increase in permeability. The mass transier is increased in the region of the cannister due to the approach of the heat pipe region.

Fig.4(f) shows the gas pressure profile for the minimum permeability of $k_{m}=3.23 \times$ $10^{-18} \mathrm{~m}^{2}$. For this low matrix permeability, the region surrounding the cannister has a constant gas pressure until the beginning of the heat pipe. The gas pressure increases sharply to a maximum value just prior to the beginning of the heat pipe. The maximum gas pressure corresponds to the boiling end of the heat pipe. An abrupt drop in gas pressure is observed at the condensation point of the heat pipe. A hump is developed in this way and this region represents the heat pipe region. The existence of a strong gas pressure gradient produces a strong gas flow away from cannister. The gas pressure remains constant beyond the heat pipe region.

Fig.4(g) depicts the gas pressure profile for the maximum permeability of $k_{m}=3.23 \times$ $10^{-15} \mathrm{~m}^{2}$. For this value, the heat pipe region is extended up to the heat source and the extent of heat pipe is larger than the previous case. Liquid saturation is obtained at the bore hole wall with temperature remaining at $100{ }^{\circ} \mathrm{C}$ in the near field. The gas pressure has the maximum value at the heat source and decreases slowly to a constant value with increasing distance from the canister and remains unchanged afterwards. This graph does not show any hump. For higher permeabilities. the gas flow is much stronger through the matrix and fracture. A small pressure gradient is sufficient to produce a strong gas flow for higher permeabilities.

Fig.4(h) and Fig.4(i) show the capillary pressure profiles for various matrix permeabilities in increasing order of magnitude. For low matrix permeabilities, the capillary pressure remains constant with its maximum value in the vicinity of the heat source. This maximum 
pressure remains constant until the beginning of the heat pipe region because the near region surrounding the cannister is in a dry out condition without any liquid saturation. This is responsible for the maximum capillary pressure in this region. The capillary pressure is suddenly dropped in the heat pipe region. Liquid saturation begins to develop in the heat pipe region and this is responsible for the sudden drop in capillary pressure. After the heat pipe region. the capillary pressure remains constant with increasing distance. This corresponds to the undisturbed region with constant liquid saturation.

The maximum capillary pressures for higher permeabilities are found at the bore hole wall. but do not remain constant in the near region as in the case of lower permeabilities. The capillary pressure gradually decreases with increasing distance away from the cannister. Some moisture is present at the bore hole wall and it increases gradually with increasing distances. This gradual increase in liquid saturation produces the gradual decrease in the capillary pressure. The capillary pressure finally attains a constant value and remains unchanged afterwards. This happens just beyond the heat pipe region and it corresponds to the undisturbed region with constant liquid saturation.

From all the above graphs and results with different permeabilities. many observations can made. The length of heat pipe increases with the increase in permeability. Also the heat pipe is shifted towards the heat source as the permeability is increased. For higher permeabilities like $k_{m}=3.2 \times 10^{-16} \mathrm{~m}^{2}$ and $k_{m}=3.210^{-15} \mathrm{~m}^{2}$, the heat pipe is extended right up to the emplacement wall. In this case. the liquid is brought into the vicinity of the cannister. This shows the significance of the matrix permeability on the performance of heat pipe in a repository. 


\subsubsection{MATRIX THERMAL CONDUCTIVITY}

The effect of thermal conductivity was tested for a range of values using the TOUGH code. For all these simulations. the difference between thermal conductivities at fully saturated conditions and fully dewatered conditions is taken approximately as 0.20 in agreement with the previous studies. The fully saturated value of the thermal conductivity was tested for a minimum value of $1.16 \mathrm{~W} / \mathrm{m}^{\circ} \mathrm{C}$ and a maximum value of $2.09 \mathrm{~W} / \mathrm{m}^{\circ} \mathrm{C}$, and for two intermediate values. The different thermal conductivities tested are shown in table 4.14 below.

Table 4.14

\begin{tabular}{|l|l|}
\hline $\begin{array}{l}\text { Run } \\
\#\end{array}$ & $\begin{array}{l}\text { Th. conductivity } \\
\left(\mathrm{W} / \mathrm{m}^{\circ} \mathrm{C}\right)\end{array}$ \\
\hline 1 & 1.16 \\
2 & 1.48 \\
3 & 1.80 \\
4 & 2.09 \\
\hline
\end{tabular}

This range is selected from the borehole studies conducted in different fractured tuffs at Yucca Mountain. Graphs are plotted as in the case of matrix permeability. Temperature and capillary pressure profiles at 1 year describe the effect of thermal conductivity. Fig.4(j) shows the temperature profiles at 1 year for different thermal conductivities used for the simulations. For the minimum thermal conductivity ialue of $1.16 \mathrm{~W} / \mathrm{m}^{\circ} \mathrm{C}$, a maximum temperature of $315^{\circ} \mathrm{C}$ is obtained and for a maximum thermal conductivity of $2.09 \mathrm{~W} / \mathrm{m}^{\circ} \mathrm{C}$, 
the corresponding maximum temperature is just $187^{\circ} \mathrm{C}$. As the thermal conductivity is increased. the maximum temperature in the near region surrounding the cannister is decreased. The near field temperatures shoot up for the lower thermal conductivity. A large thermal conductivity requires small temperature gradient and small thermal conductivity needs higher temperature gradient to produce the same heat flux.

It can be seen that the increase in thermal conductivity causes a slight increase in the liquid saturation. This is due to the reduction in the dry out region around the bore hole wall as a result of the reduced temperatures. The condensation point is shifted towards the heat source with the increase in thermal conductivity.

Fig.4(k) shows the behavior of capillary pressure at 1 year for different thermal conductivities tested. The capillary pressure profiles behave in the same manner with one difference. The maximum capillary pressure region near the bore hole wall decreases in length with increase in thermal conductivity due to the reduction of the dry out region with the increase in thermal conductivity. The capillary pressure has the maximum value in the near region surrounding the cannister and it is maintained at that value for some distance in the near region. A large drop in the capillary pressure occurs at the beginning of the heat pipe region. A constant capillary pressure is maintained in the undisturbed region just after the heat pipe region.

\subsubsection{MATRIX TORTUOSITY}

The reference value for the tortuosity is taken as 0.40 [Bear et al.. 1968]. Based upon this reference value. some upper and lower values are assigned for the tortuosity factor for the different simulations to study the tortuosity effect on the heat pipe performance. The tortuosity values tested are given in table 4.15 below. 
Table 4.15

\begin{tabular}{|l|l|}
\hline Run \# & Tortuosity \\
\hline 1 & 0.50 \\
2 & 0.40 \\
3 & 0.20 \\
4 & 0.10 \\
\hline
\end{tabular}

From the results of the computer simulations with different tortuosities, it was found that the tortuosity does not have any impact on the heat pipe effect and the point of condensation. From these results, it was observed that neither the matrix nor the fracture undergoes any significant changes in their temperature or liquid saturation values by changing the tortuosity values of the rock matrix. The temperatures at some selected distances are given in table 4.16 below.

Table 4.16

\begin{tabular}{|l|l|l|l|l|l|l|l|l|}
\hline \multirow{2}{*}{ Run } & \multirow{2}{*}{ Tortuosity } & \multicolumn{6}{|c|}{ Temperature in ${ }^{\circ} \mathrm{C}$ at certain distances } \\
\cline { 2 - 9 } & factor & $.27 \mathrm{~m}$ & $.61 \mathrm{~m}$ & $.71 \mathrm{~m}$ & $.84 \mathrm{~m}$ & $.97 \mathrm{~m}$ & $1.13 \mathrm{~m}$ & $1.52 \mathrm{~m}$ \\
\hline 1 & 0.50 & 144.0 & 100.8 & 100.5 & 100.2 & 99.9 & 90.3 & 73.9 \\
\hline 2 & 0.40 & 144.2 & 100.7 & 100.3 & 100.2 & 99.8 & 90.2 & 74.0 \\
\hline 3 & 0.30 & 144.1 & 100.7 & 100.4 & 100.3 & 99.8 & 90.4 & 74.2 \\
\hline 4 & 0.10 & 144.0 & 100.8 & 100.4 & 100.2 & 99.9 & 90.4 & 74.0 \\
\hline
\end{tabular}




\subsubsection{MATRIX POROSITY}

The effect of porosity is tested for a set of values using the TOUGH code. These values are taken from [Peters et al.. 1984] and correspond to different fractured tuffs at Yucca Mountain. The tested values of porosity in this study are given in table 4.17 below.

Table 4.17

\begin{tabular}{|l|l|}
\hline Run \# & Porosity \\
\hline 1 & 0.05 \\
2 & 0.10 \\
3 & 0.15 \\
4 & 0.25 \\
\hline
\end{tabular}

Temperature values corresponding to different porosities after 51 days are shown shown in table 4.18 below. As porosity increases. the temperature at any particular point in the matrix decreases slightly, but the change in temperature and liquid saturation is so small that the effect of porosity on the heat pipe performance can be neglected. After one year, the porosity did not make even minor changes in the results. 
Table 4.18

\begin{tabular}{|l|l|l|l|l|l|l|l|l|l|}
\hline \multirow{2}{*}{ Run } & \multirow{2}{*}{ Variable } & \multicolumn{7}{|c|}{ Temperature in ${ }^{\circ} \mathrm{C}$ at certain distances } \\
\cline { 2 - 10 } & $(\mathrm{n})$ & $.27 \mathrm{~m}$ & $.61 \mathrm{~m}$ & $.71 \mathrm{~m}$ & $.84 \mathrm{~m}$ & $.97 \mathrm{~m}$ & $1.13 \mathrm{~m}$ & $1.52 \mathrm{~m}$ & $5.52 \mathrm{~m}$ \\
\hline 1 & 0.05 & 147.2 & 104.1 & 100.5 & 100.2 & 99.5 & 92.4 & 75.3 & 28.8 \\
\hline 2 & 0.10 & 146.7 & 103.7 & 100.4 & 100.0 & 99.1 & 90.0 & 74.5 & 28.2 \\
\hline 3 & 0.15 & 145.8 & 102.8 & 100.1 & 99.9 & 98.8 & 89.4 & 74.0 & 28.1 \\
\hline 4 & 0.25 & 144.7 & 102.0 & 100.0 & 99.8 & 98.2 & 89.1 & 73.8 & 28.0 \\
\hline
\end{tabular}

\subsubsection{MATRIX SPECIFIC HEAT}

The specific heat $\left(C_{p}\right)$ of the rock matrix has some effect on the heat pipe performance, but it is not as important compared as the permeability or the thermal conductivity. The specific heat values tested were in the range of $C_{p}=400 \mathrm{~J} / \mathrm{kg}^{\circ} \mathrm{C}$ and $C_{p}=1250 \mathrm{~J} / \mathrm{kg}^{\circ} \mathrm{C}$. This range of values corresponds to different fractured tuffs found in Yucca Mountain [Peters et al.. 1984].

Table 4.19

\begin{tabular}{|l|l|}
\hline Rin & $\begin{array}{l}\text { Specific heat } \\
\left(J / \text { kg }^{\circ} \mathrm{C}\right)\end{array}$ \\
\hline 1 & 600 \\
2 & 768 \\
3 & 1000 \\
4 & 1250 \\
\hline
\end{tabular}


Fig.4(1) shows the temperature profiles at 1 year for different specific heat tested. The flatter region of the graph represents the heat pipe region. The maximum temperature obtained after 1 year for the minimum specific heat was $208{ }^{\circ} \mathrm{C}$ while the corresponding temperature for the maximum specific heat was $192^{\circ} \mathrm{C}$. Large specific heat, produces reduced temperatures and high liquid saturations in the region close to the bore hole wall so that favourable two phase condition exists in the near field.

The temperature profiles at 51 days were compared to the temperature distributions at 1 year for the same heat capacities. The specific heat produced changes in temperature and liquid saturations after 51 davs, but the simulations at 1 year showed no change in results associated with the specific heat. This is due to the development of the thermodynamic equilibrium at one year. The effect of specific heat is significant only on the transient. Once the equilibrium is acheived, the specific heat will not have any effect on the heat pipe performance.

\subsection{SENSITIVITY ANALYSIS}

The exact influence of different parameters on the length of the heat pipe is found by computing the sensitivities of the heat pipe length with respect to each of these parameters. Each variable is tested first with their mean values. The mean values of permeability, thermal conductivity, porosity and specific heat are taken as $3.2 \times 10^{-17} \mathrm{~m}^{2}, 1.80 \mathrm{~W} / \mathrm{m}{ }^{\circ} \mathrm{C}$, 0.14 and $1000 \mathrm{~J} / \mathrm{kg}^{\circ} \mathrm{C}$ respectively. The length of the heat pipe for the mean value is determined. Then two more simulations are carried out for the same variable with $x+\delta$ and $x-\delta$ where $\mathrm{x}$ is the mean value of the variable and $\delta$ is the variation. The length of the heat pipe for these two values is compared with the length obtained for the mean value 
and the change in lengtin for these two values from the mean value is computed. Then the sensitivity $S$ is calculated as given below.

$$
S=\frac{1}{F} \frac{\partial F}{\partial X_{i}}
$$

where $S$ is the sensitivity, $F$ is any property for which the sensitivity is to be determined with respect to any paraneter $\mathrm{X}$.

The sensitivity of the heat pipe length for all the variables is calculated as described above. The parameters influencing the length of heat pipe and their exact influence are found by comparing all these sensitivities. The computed sensitivities are given in table 4.20 below. 
Table 4.20

\begin{tabular}{|c|c|c|c|c|c|}
\hline $\begin{array}{l}\text { Run } \\
\#\end{array}$ & $\begin{array}{l}\text { Testing } \\
\text { variable }\end{array}$ & $\begin{array}{l}\text { Numerical } \\
\text { value }\end{array}$ & $\begin{array}{l}\text { Length of } \\
\text { heat pipe( } \mathrm{m})\end{array}$ & $\begin{array}{l}\text { Change in } \\
\text { length }(m)\end{array}$ & $\begin{array}{l}S \\
(\%)\end{array}$ \\
\hline 1 & $k\left(m^{2}\right)$ & $3.2 e-1 i$ & 1.17 & - & - \\
\hline 2 & $n$ & $3.2 \mathrm{e}-18$ & 0.66 & -0.51 & 44 \\
\hline 3 & $\mapsto$ & $3.2 \mathrm{e}-16$ & 2.06 & +0.89 & 76 \\
\hline 4 & porosity & 0.14 & 0.72 & - & - \\
\hline 5 & 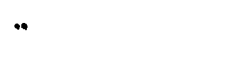 & 0.18 & 0.82 & +0.10 & 13 \\
\hline 6 & $\cdots$ & 0.10 & 0.66 & -0.06 & 8 \\
\hline 7 & $\mathrm{~K}\left(W / \mathrm{m}^{\circ} \mathrm{C}\right)$ & 2.07 & 0.72 & - & - \\
\hline 8 & 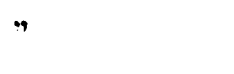 & 2.23 & 0.51 & -0.21 & 29 \\
\hline 9 & 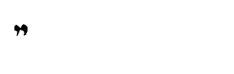 & & 0.90 & +0.18 & 25 \\
\hline 10 & $C_{p}\left(J / k g{ }^{\circ} C\right)$ & 1000 & 0.72 & - & - \\
\hline 11 & $"$ & 1200 & 0.78 & +0.06 & 9 \\
\hline 12 & 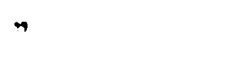 & 800 & 0.78 & +0.06 & 9 \\
\hline
\end{tabular}

From this table, the highest sensitivity is obtained for the matrix permeability. Sensitivity is also higher for thermal conductivity. Sensitivities are found to be small for porosity and specific heat. These two parameters do not affect the heat pipe region considerably and the effect of specific heat is negligibly small. The most important parameters affecting the extent and length of heat pipe are the matrix permeability and the matrix thermal conductivity.

The sensitivity of the temperature at the bore hole wall to the matrix permeability and 
the matrix thermal conductivity are calculated and presented in table 4.21 below.

Table 4.21

\begin{tabular}{|l|l|l|l|l|l|}
\hline Run & $\begin{array}{l}\text { Testing } \\
\text { variable }\end{array}$ & value & $\begin{array}{l}\text { Vumerical } \\
\left({ }^{\circ} \mathrm{C}\right)\end{array}$ & $\begin{array}{l}\text { Temperature } \\
\left.\text { change }{ }^{\circ} \mathrm{C}\right)\end{array}$ & $\begin{array}{l}\text { Temperature } \\
(\%)\end{array}$ \\
\hline 1 & $\mathrm{k}\left(\mathrm{m}^{2}\right)$ & $3.2 \times 10^{-17}$ & 142.7 & - & - \\
2 & $\eta$ & $3.2 \times 10^{-18}$ & 166.5 & +23.8 & 17 \\
3 & $\eta$ & $3.2 \times 10^{-16}$ & 102.0 & -40.7 & 29 \\
\hline 4 & $\mathrm{~K}\left(W / m^{\circ} \mathrm{C}\right)$ & 1.80 & 149.6 & - & - \\
5 & $\cdots$ & 1.50 & 168.0 & +18.4 & 13 \\
6 & $\eta$ & 2.10 & 133.3 & -16.3 & 11 \\
\hline
\end{tabular}

From table 4.21, it is obvious that the temperature is sensitive to both the matrix permeability and the thermal conductivity, but the temperature is more sensitive to the matrix permeability than the matrix thermal conductivity.

\subsection{CONVECTIVE \& CONDUCTIVE HEAT TRANSFER}

The Nusselt number at any point in the heat pipe region is defined as the ratio of convective heat transfer to the conductive heat transfer at that particular point. Total heat transferred at the heat pipe region is obtained from the computer simulations. The heat conducted is calculated by Fourier's law.

$$
Q_{c d}=\pi A \frac{d T}{d x}
$$


where $Q_{c d}$ is the heat conducted in Watts. $K$ is the thermal conductivity at that point, $A$ is the interiacial area of the two elements across which heat is conducted and $\frac{d T}{d x}$ is the temperature gradient where $\mathrm{dx}$ is the center to center distance of the two elements involved in the heat transfer and $d T$ is the difference in temperature. The thermal conductivity $K$ is determined by using the following relationship[Somerton et al.. 1973].

$$
K=K_{d r}+S_{l}\left(K_{w e}-K_{d r}\right)
$$

where $S_{l}$ is the average liquid saturation of the two elements. The heat convected in Watts is obtained as

$$
Q_{c v}=Q-Q_{c d}
$$

where $Q$ is the total heat transfer in Watts which is obtained directly from the computer simulations. Now the Nusseit number is defined by

$$
N u=\frac{Q_{c v}}{Q_{c d}}
$$

The Nusselt numbers for the fracture and the matrix are calculated. The overall Nusselt number considering the heat transfer through both the fracture and the matrix is also calculated. The computed Nusselt numbers and the ratio $\frac{Q_{f}}{Q_{m}}$ are given in the table shown below. $. V u_{f}, \nu u_{m}$, and.$V u_{t}$ represent the fracture $N$ usseit number, matrix Nusselt number and overall Nusseit number respectively. $Q$, and $Q_{m}$ are the total heat flow through the fracture and the matrix respectively. These Nusselt numbers and the ratio $\frac{Q_{f}}{Q_{m}}$ are calculated at a distance of 1.75 meters from the center line of the cannister. This particular distance corresponds to element \#13 which was common in the heat pipe region for all the 
simulations.

Table 4.22

\begin{tabular}{|c|c|c|c|c|c|c|c|c|}
\hline \multirow{2}{*}{$\begin{array}{l}\text { Testing } \\
\text { variable }\end{array}$} & \multirow{2}{*}{$\begin{array}{l}\text { Numerical } \\
\text { value }\end{array}$} & \multicolumn{7}{|c|}{ Nusseit \#'s and their sensitivities at the heat pipe region } \\
\hline & & $N u_{f}$ & $\mathrm{~S}(\%)$ & $N u_{m}$ & $S(\%)$ & $N u_{t}$ & $\mathrm{~S}(\%)$ & $\frac{Q_{1}}{Q_{m}}$ \\
\hline $\mathbf{k}\left(m^{2}\right)$ & $3.2 \mathrm{e}-17$ & 2943 & - & -3.68 & - & 21.23 & - & -9.36 \\
\hline$n$ & $3.2 \mathrm{e}-18$ & 3818 & 30 & -1.50 & 59 & 9.42 & 55 & -12.16 \\
\hline$"$ & $3.2 \mathrm{e}-16$ & 4812 & 63 & -6.68 & 81 & 36 & 70 & -6.59 \\
\hline porosity & 0.14 & 3890 & - & -3.72 & - & 22.18 & - & -9.57 \\
\hline$n$ & 0.18 & 3847 & 1.1 & -3.69 & 6 & 21.69 & 2.2 & -9.80 \\
\hline$n$ & 0.10 & 3946 & 1.4 & -3.69 & 6 & 21.26 & 4.3 & -9.35 \\
\hline $\mathrm{K}\left(W / m^{\circ} C\right)$ & 2.07 & 2763 & - & -2.60 & - & 14.83 & - & -10.99 \\
\hline$n$ & 2.23 & 2255 & 18 & -2.81 & 8 & 16.75 & 13 & -10.90 \\
\hline$n$ & 1.91 & 3112 & 13 & -2.99 & 15 & 16.10 & 9 & -9.66 \\
\hline$C_{p}\left(J / k g^{\circ} C\right)$ & 1000 & 2932 & - & -3.12 & . & 17.61 & - & -9.86 \\
\hline$\pi$ & 1200 & 2924 & 1.1 & -2.97 & 5 & 16.90 & 4 & -8.61 \\
\hline$n$ & 800 & 2915 & 1.4 & -3.24 & 4 & 18.13 & 3 & -9.63 \\
\hline
\end{tabular}

A negative sign in table 4.22 for $\frac{Q_{f}}{Q_{m}}$ means that the flow of heat through the fracture and the matrix are in opposite directions. The net flow of heat in the matrix will be the sum of the heat conducted away from the cannister. the heat convected by the gas away from the cannister and the heat convected by the liquid towards the cannister. In all cases, there is a net flow of heat towards the cannister through the matrix so that the heat convection by 
liquid flow will be greater than the sum of conduction and the heat transported by the gas flow. The flow of heat in the fracture is primarily by convection and it is away from the heat source. For the overall system. the net flow of heat is away from the heat source, so it is always positive. A negative sign in the case of Nusselt number means that the convection and the conduction are in opposite directions. From the above table, the sensitivities of $N u_{f}, N u_{m}$ and $N u_{t}$ are found to be higher for the matrix permeability and the thermal conductivity compared to the rest of the parameters.

The ratio of the fracture heat flow to the matrix heat flow $\left(\frac{Q_{1}}{Q_{m}}\right)$ gave some interesting results for the matrix permeability. The change in this ratio is very high for the matrix permeability compared to other parameters. This is due to the increase in convective heat transfer. The net heat flow in the matrix is due to the convection. At change in matrix permeability produced a proportional change in $\frac{Q_{1}}{Q_{m}}$. In other words, the ratio $\frac{Q_{1}}{Q_{m}}$ is directly proportional to the matrix permeability.

The Nusseit numbers and their sensitivities do not change considerably for the matrix porosity and the specific heat. These observations are in full agreement with the sensitivity analysis of the heat pipe length. $. V u_{f}, V u_{m}, V u_{t}$ and their sensitivities are found to be the maximum in the case of a maximum permeability of $3.2 \times 10^{-16} \mathrm{~m}^{2} . N u_{f}$ values in all cases are very large compared to all the matrix Nusseit numbers and the overail Nusselt numbers.

For all the parameters tested. the fracture Nusselt numbers are in the order of thousands and the average $N u_{f}$ is around 3000 . This indicates that the convection is dominating over conduction in the fracture and conductive heat transfer in the fracture can be almost neglected. The matrix . Nusselt numbers are extremely small compared to $N u_{f}$ and the 
average $N u_{m}$ of the different simuiations is around 3 oniy. This means both convection and conduction are important even though convection is stronger compared to conduction. The overall Nusseit numbers are large compared to $N u_{m}$, still small compared to $N u_{f}$. The average value of $N u_{t}$ is approximately 10. Here also. convection is predominant compared to conduction. But the conductive heat transfer cannot be neglected in the overall system.

The Nusselt number serves as a measure of the strength of the heat pipe. A heat pipe is considered to be stronger if the convection is dominating over conduction significantly. In table 4.22. the largest Nusselt number is obtained for the maximum permeability tested and the heat pipe is very strong in this case. The ratio $\frac{Q_{1}}{Q_{m}}$ is always greater than one and around 10 in most of the cases. These results show that the heat flow is mainly due to convection through the fracture which is quite expected in the heat pipe region.

\subsection{MATRIX MODELING RESULTS}

Some important results were obtained from the matrix modeling. The effects of various parameters on the heat pipe performance are examined in detail in the matrix modeling. They include the strength and extent of the heat pipe, the temperature profiles, the fracturematrix equilibrium and the shift in condensation point. The importance of convective heat transfer and the type of heat transfer mechanism in the fracture and the matrix are also discussed in detail.

The strength and the extent of the heat pipe is greatly influenced by some of the tested parameters. Matrix permeability and matrix thermal conductivity are the two parameters that influence the heat pipe region significantly. Matrix permeability is the most important parameter. Large matrix permeabilities increase the heat pipe length. Large permeabili- 
ties also increase the strength of the heat pipe by increasing the convective heat transfer. Porosity, specific heat and tortuosity do not cause significant changes in the length and the strength of the heat pipe.

The temperature distribution around the cannister is affected considerably by some of the parameters. Large permeabilities reduce the temperatures in the near region around the cannister and eliminate the dry out region surrounding the cannister. Two phase conditions exist up to the bore hole wall, which remains at or near $100^{\circ} \mathrm{C}$. Temperature profiles are slightly changed by the specific heat and porosity during the initial stages of the transient. but become unaffected after one year. Tortuosity does not affect the temperature distribution in the repository.

Some of the matrix parameters shift the heat pipe region towards the cannister. An increase in permeability shifts the heat pipe region and the condensation point towards the cannister. Changes in porosity, specific heat and tortuosity do not shift the heat pipe region or condensation point.

Sensitivity analysis showed significance of the parametric effect on convective heat transfer in the heat pipe region. The heat flow is mainly due to convection through the fracture in all cases. Matrix permeability is the most sensitive parameter that influences the convective heat transfer in a fractured medium. The maximum convective effect was found for the highest permeability, due to the application of a heat source. Nusselt numbers reveal the dominance of convection over conduction. Thermal conductivity also effects the convective heat transfer. but it is not as significant as matrix permeability. Other parameters do not have much effect on the type of heat transfer mechanism in the fracture and the matrix.

Results of the matrix modeling can be expressed in terms of the energy transport and 
mass conservation equations. Different matrix properties tested have different effects on the mass and energy equations.

Referring to equation 3.1. the basic mass and energy balance equations are expressed as shown below.

$$
\frac{\partial}{\partial t} \int_{V_{n}} W^{(h)} d v=\int_{\Gamma_{n}} F^{(h)} \cdot \hat{n} d \Gamma+\int_{V_{n}} q^{(h)} d v
$$

$\int_{\Gamma_{n}} \overrightarrow{F^{(h)}} \cdot \hat{n} d \Gamma$ represents the surface flux and $\frac{\partial}{\partial t} \int_{V_{n}} M^{(h)} d v$ represent the mass rate of change in the above equation.

The heat flux term shown below consists of conductive and convective heat transfer effects, respectively.

$$
\overrightarrow{F^{h}}=\left[-K^{\nabla} \nabla T\right]+\left[h_{l}^{w} \overrightarrow{F_{l}^{w}}+h_{l}^{a} \vec{F}_{l}^{a}+h_{g}^{w} \overrightarrow{F_{g}^{w}}+h_{g}^{a} \vec{F}_{g}^{a}\right]
$$

The mass flux in each phase is given by

$$
\overrightarrow{F^{(h)}}=-k \frac{k_{r \beta}}{\mu_{\beta}} \rho_{\beta} X_{\beta}^{(h)}\left(\nabla P_{\beta}-\rho_{\beta} \vec{g}\right)-D_{v a} \rho_{\beta} \nabla X_{\beta}^{(h)}
$$

$3=6, g$ represent the liquid and gas phases in the above equations.

The heat accumulation term consists of rock and fluid properties and is given by

$$
u^{(h)}=(1-b) \rho_{R} C_{R} T+\phi\left(S_{\mid} \rho_{l} u_{\mid}+S_{\jmath} \rho_{g} u_{g}\right)
$$

The parameters in the heat flux term and the parameters in the heat accumulation term influence the heat pipe performance in different ways. The parameters in the flux term are the absolute permeability $(k)$, the relative permeabilities $\left(k_{r+\beta}\right)$ the viscosities $\left(\mu_{\beta}\right)$, the 
densities $\left(\rho_{\beta}\right)$ and the pressure gradients $\left(\nabla P_{\beta}\right)$. The parameters in the heat accumulation term are the porosity. the heat capacity and the rock density.

The effect of the heat accumulation term is on the transient. It is only important for the manner in which the thermodynamic equilibrium is established. Once the equilibrium is reached, the time derivative disappears so that the results remain unchanged. Simulations were done for different porosities at 51 days and one year. The temperatures at 51 days for various porosities showed some differences. The equilibrium is not established at this time. The temperatures at one year did not differ for various porosities used for the simulations. The equilibrium was achieved by the end of one year so that the parametric effect is vanished at this time. The specific heat produced similar results. The effect of these two parameters on the heat pipe performance after equilibrium is minimal.

The flux terms in the above equations affect the heat pipe performance in a totally different way. The flux terms affect the final steady state. An increase in absolute permeability produces an increase in mass flux. This increases the convective heat transfer. Thermal conductivity also produces changes in heat flux. An increase in thermal conductivity increases the heat flux for a given temperature gradient. This is responsible for the reduced temperatures in the region near the waste package for the higher thermal conductivities tested. Tortuosity is also a flux parameter, but its effect is negligible. 


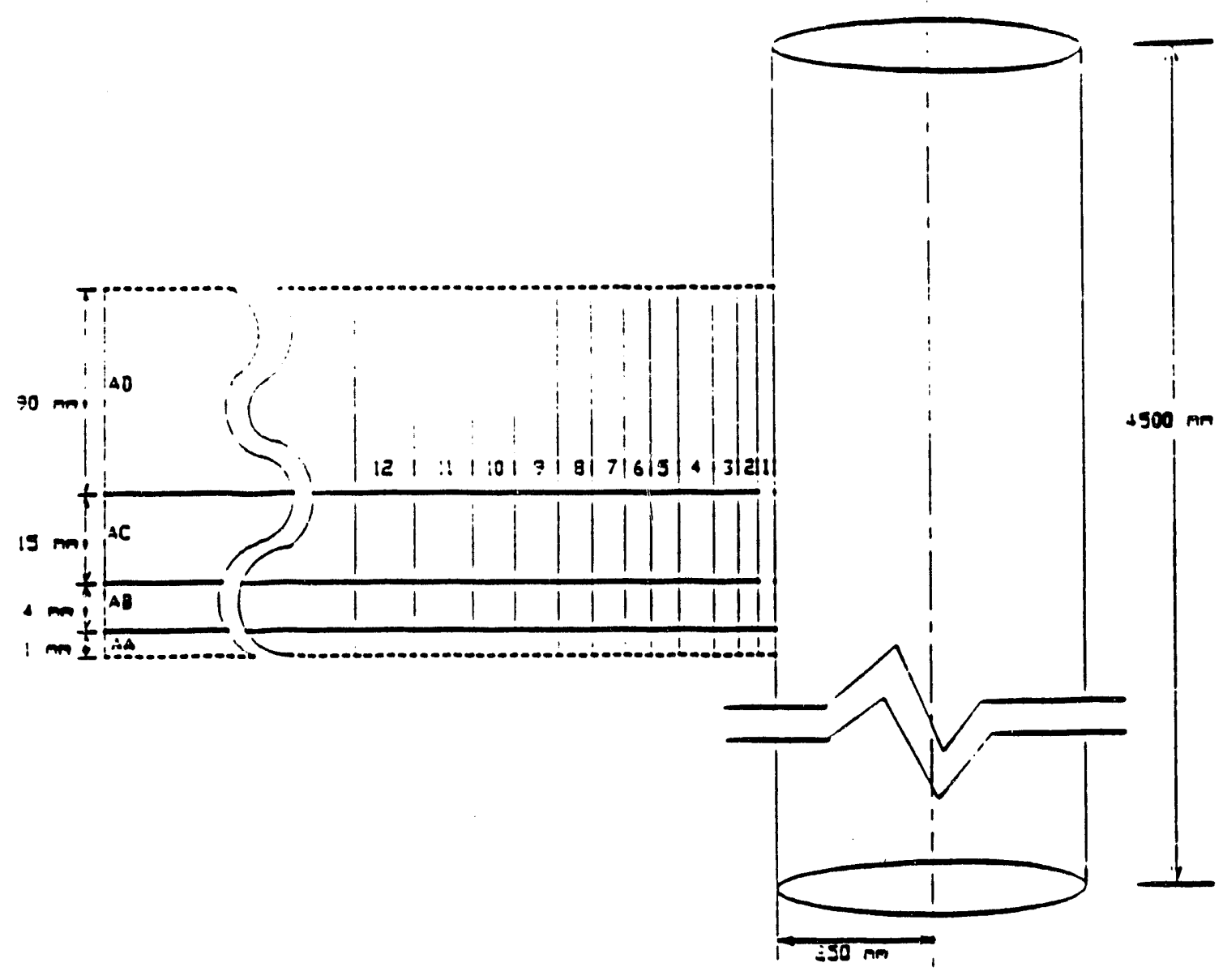

Figure 4(a): Geometry of the system modeled 


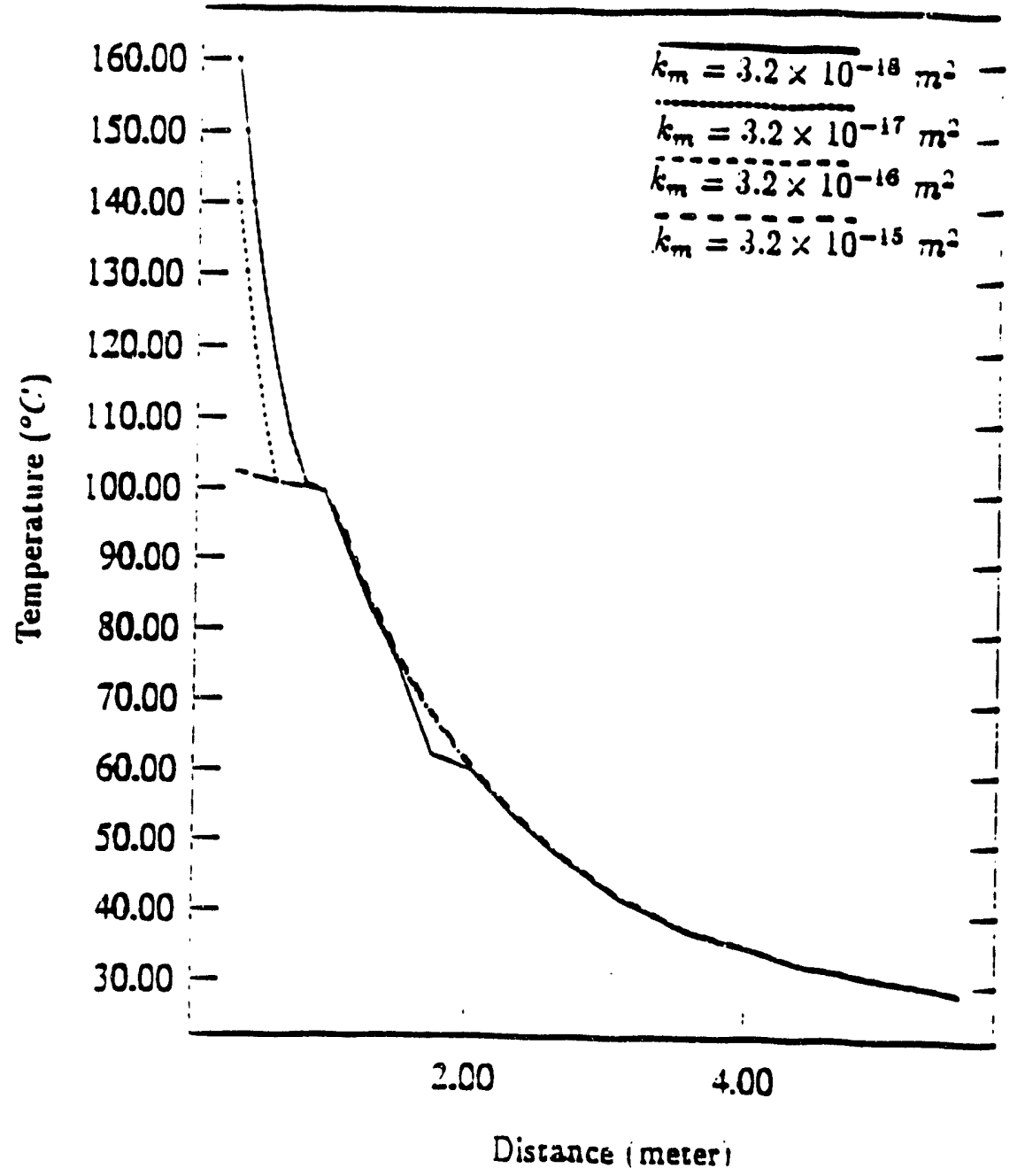

Figure 4(b): Temperature profiles for different permeabilities at 51 davs 


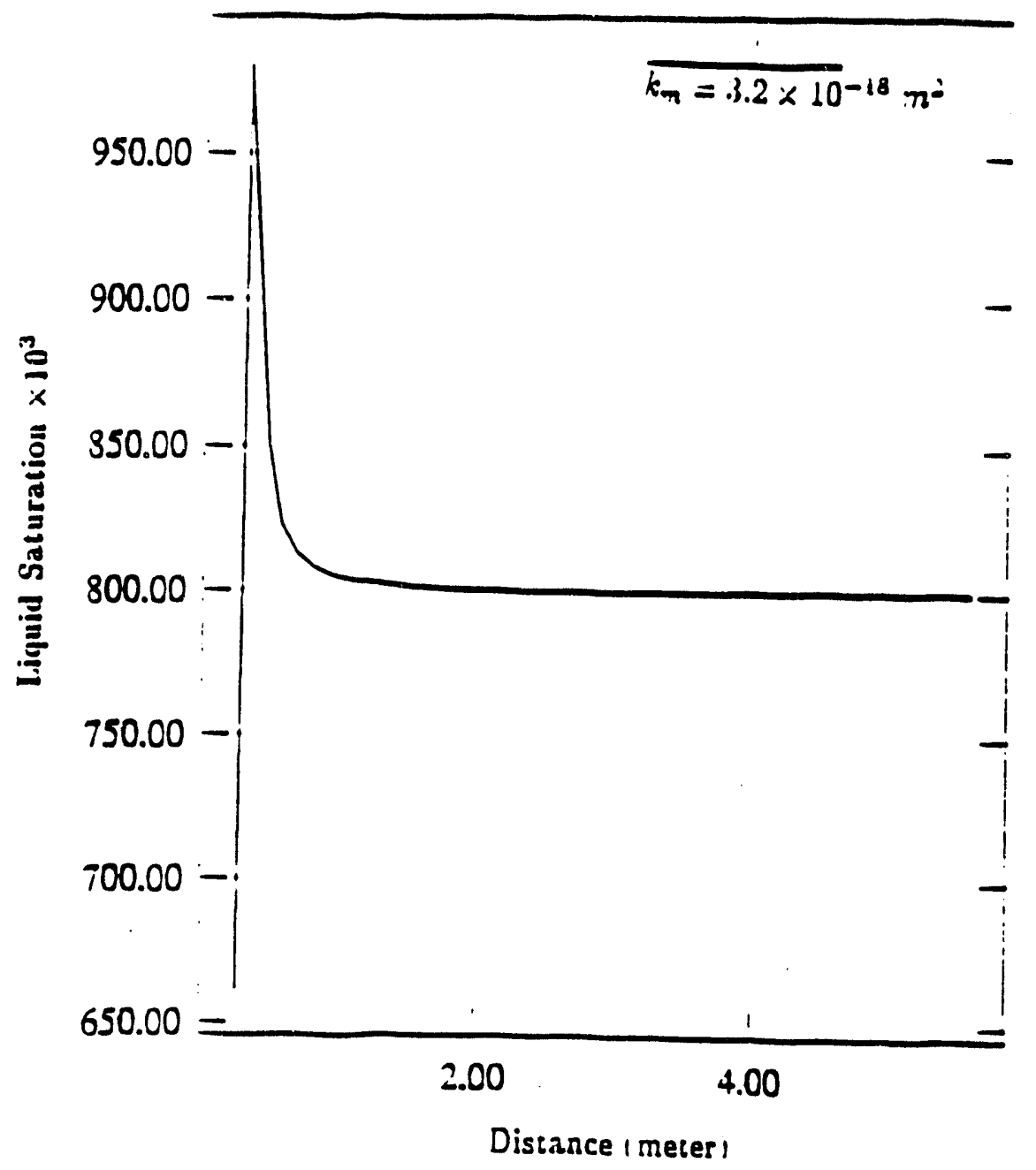

Figure 4(c): Liquid saturation profile for $k_{m}=3.2 \times 10^{-18} \mathrm{~m}^{2}$ at 51 days 


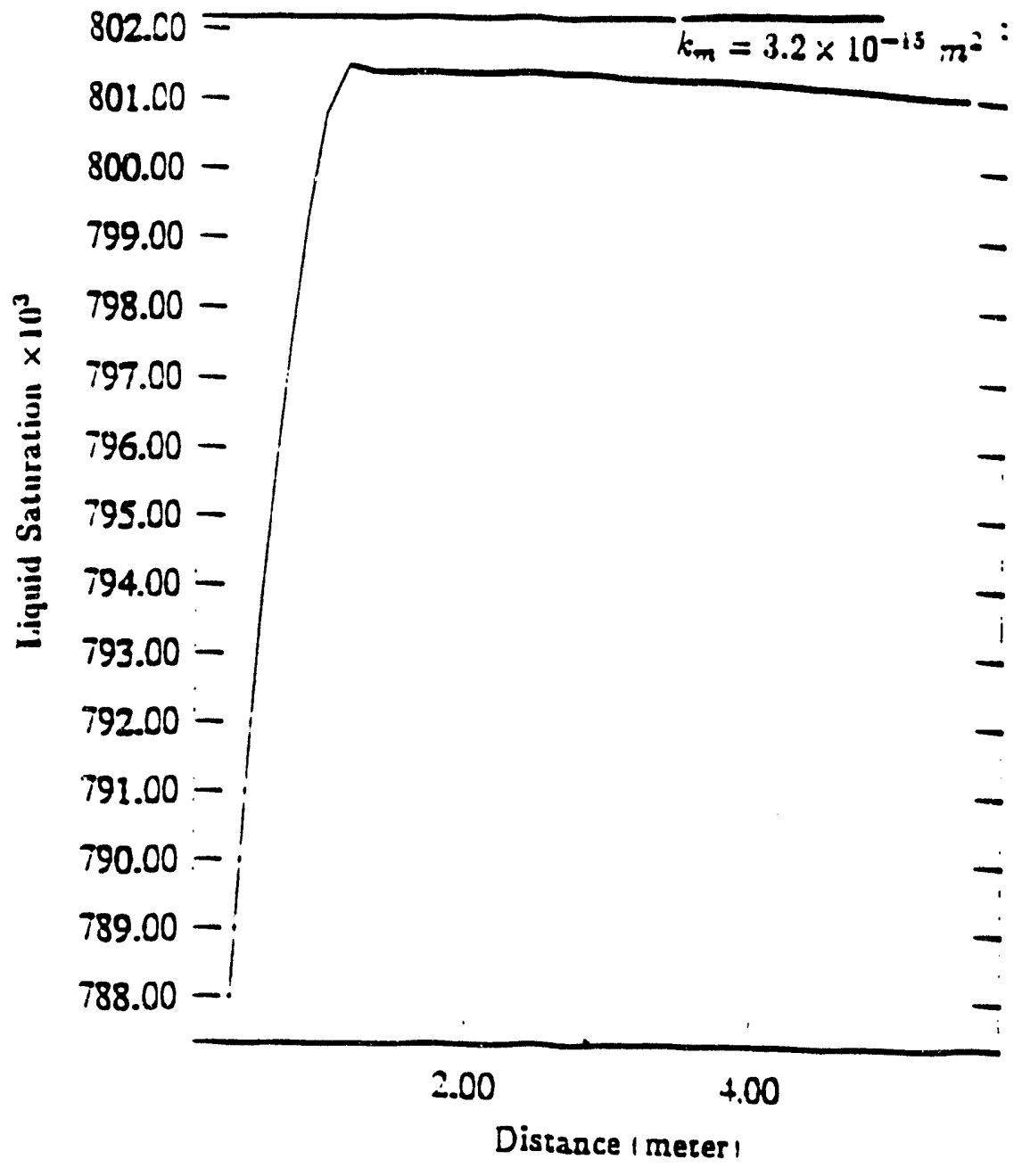

Figure $4(\mathrm{~d})$ : Liquid saturation profile for $k_{m}=3.2 \times 10^{-15} \mathrm{~m}^{2}$ at 51 days 


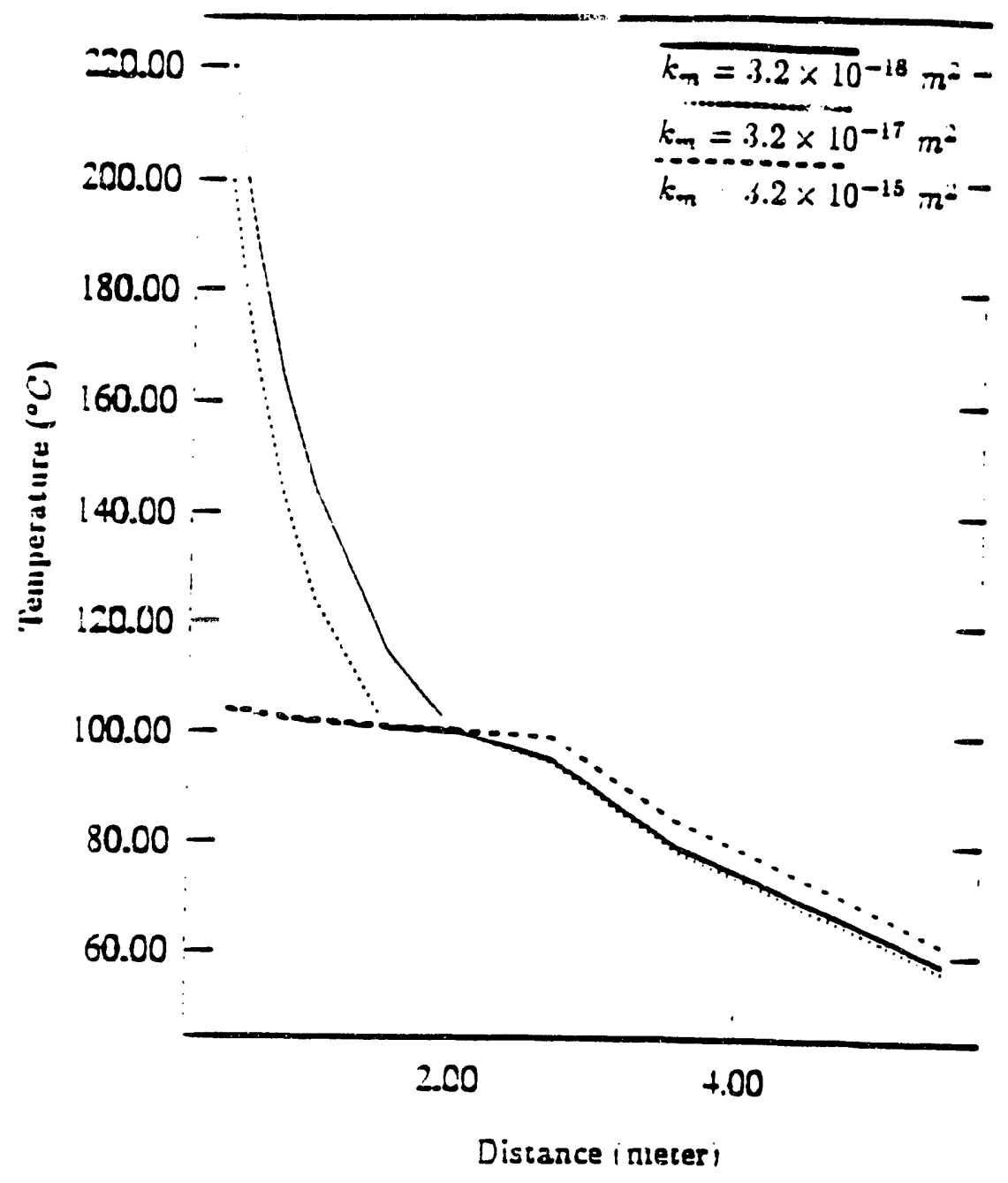

Figure 4(e): Temperature pron̂les for different permeabilities at 1 year 


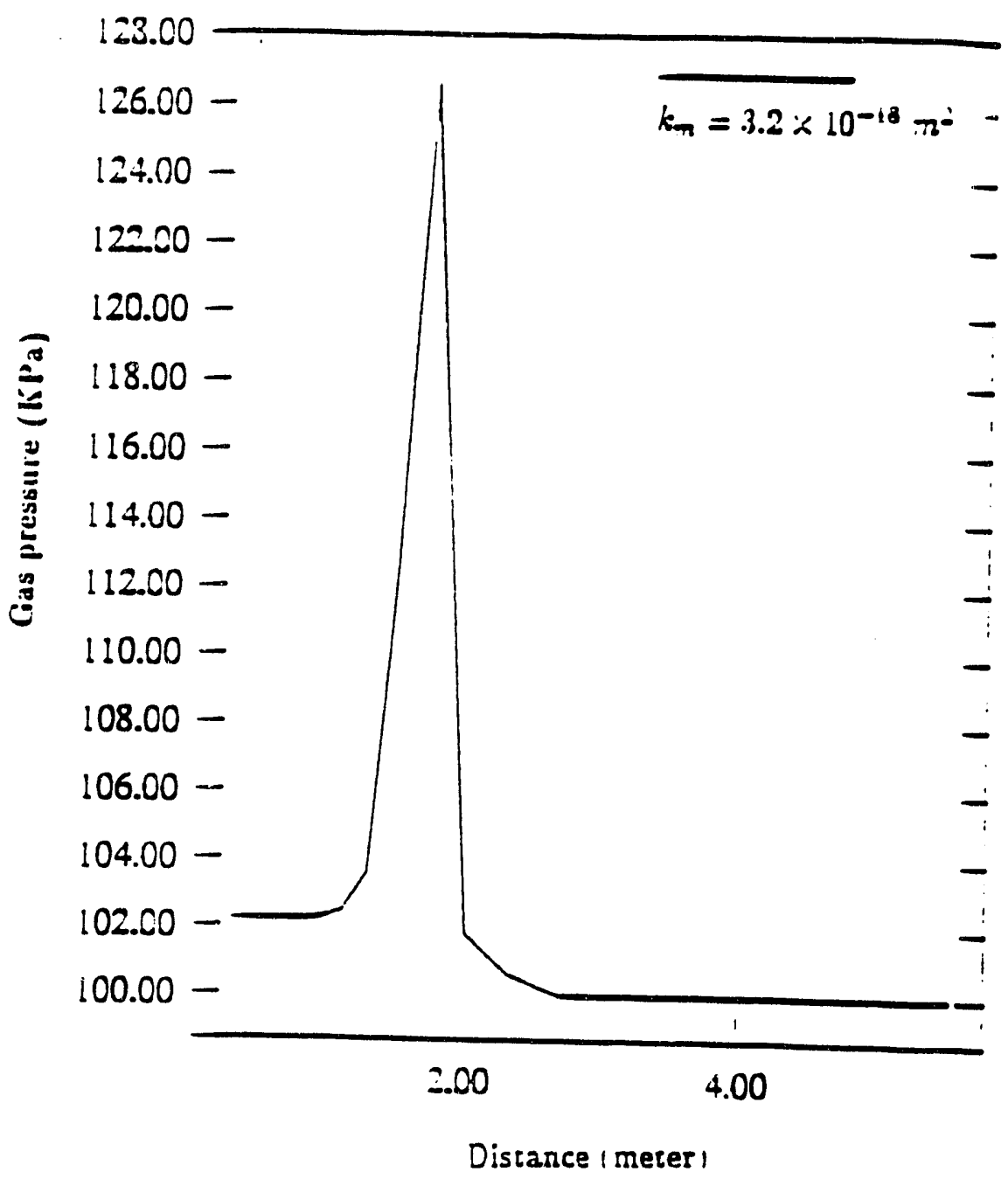

Figure 4(f): Gas pressure profile at 1 year for $k_{m}=3.2 \times 10^{-18} \mathrm{~m}^{2}$ 


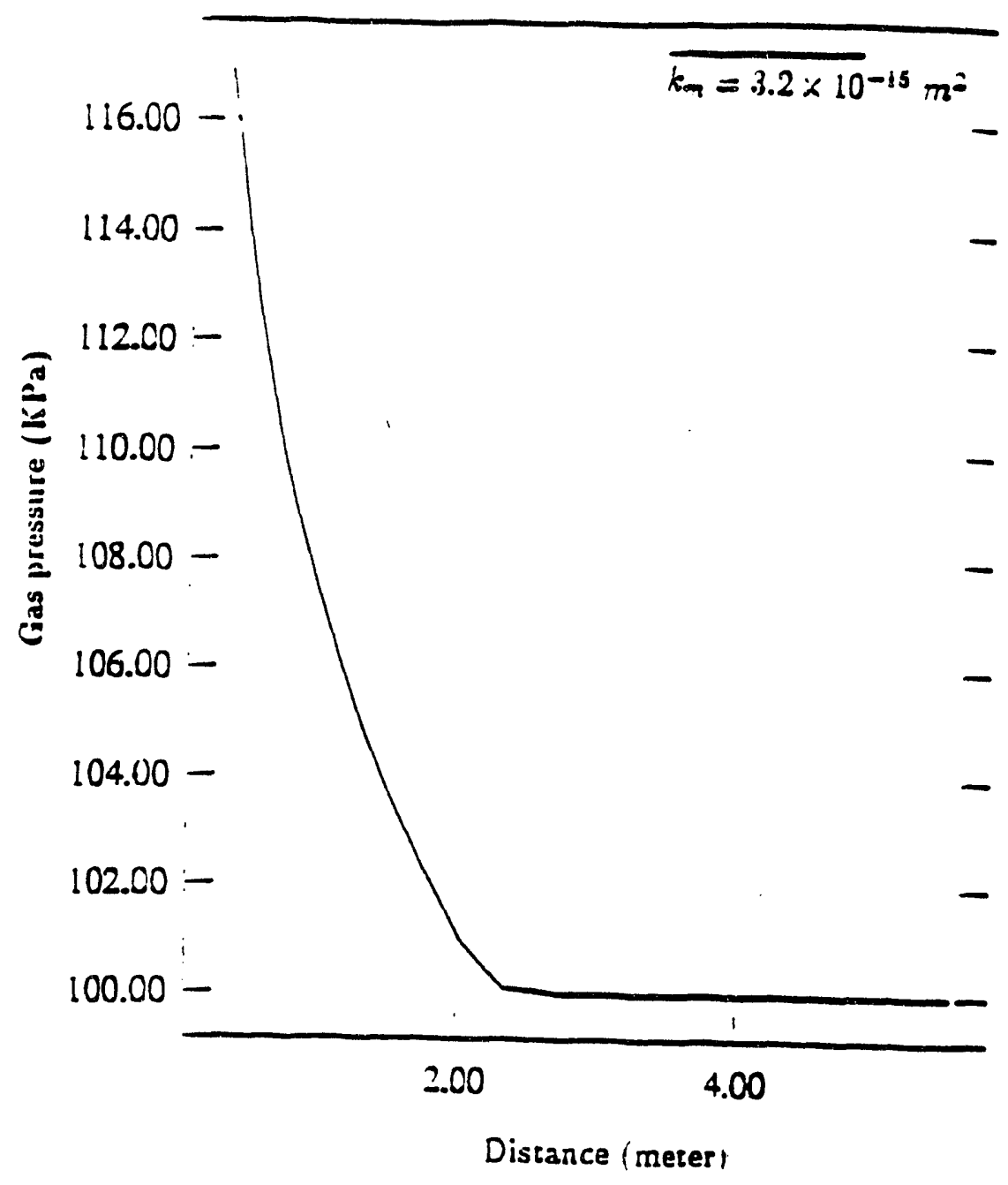

Figure $4(\mathrm{~g})$ : Gas pressure profile at 1 year for $k_{m}=3.2 \times 10^{-15} \mathrm{~m}^{2}$ 


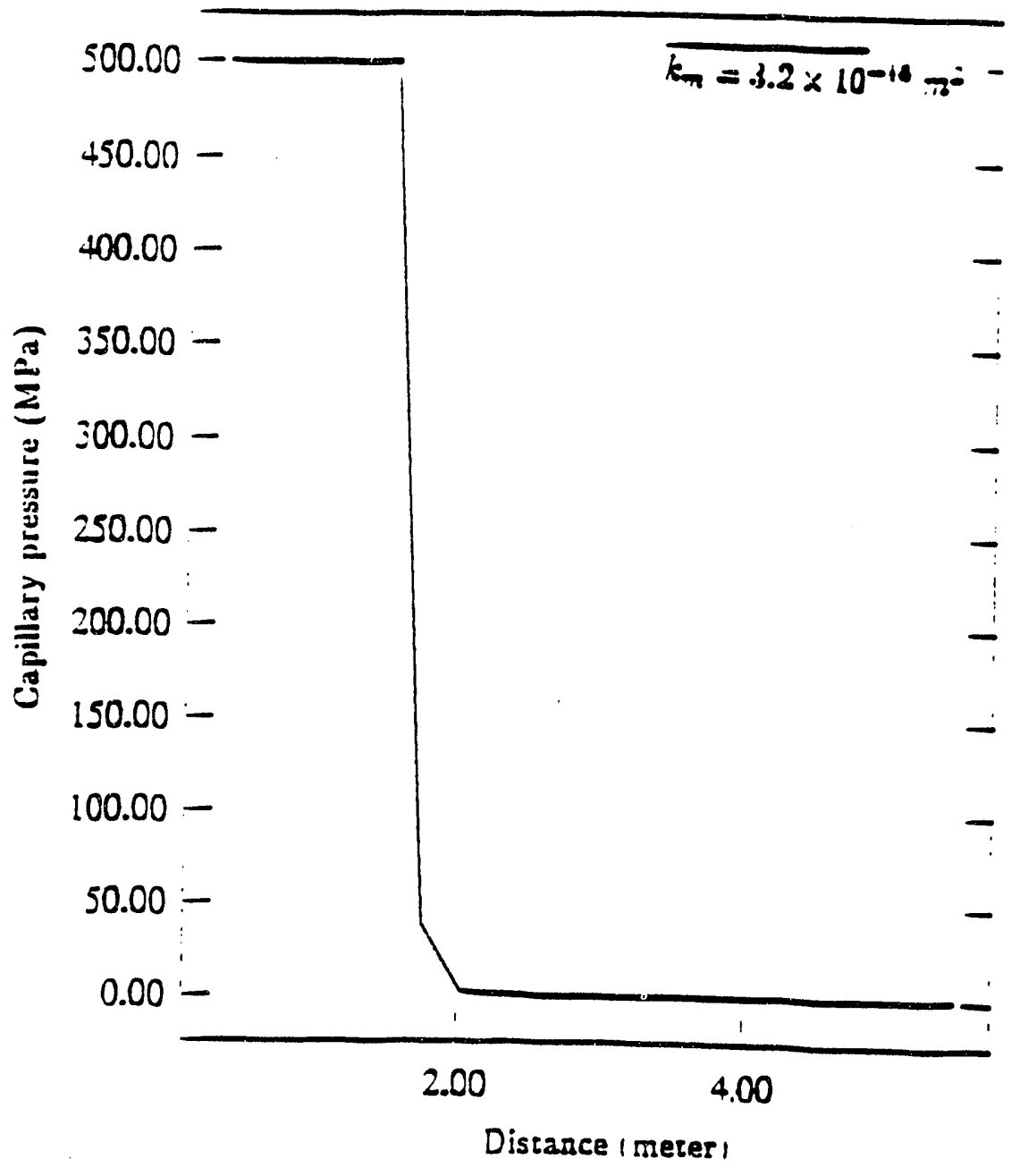

Figure $4(\mathrm{~h})$ : Capiilary pressure profile at 1 year for $k_{m}=3.2 \times 10^{-18} \mathrm{~m}^{2}$ 


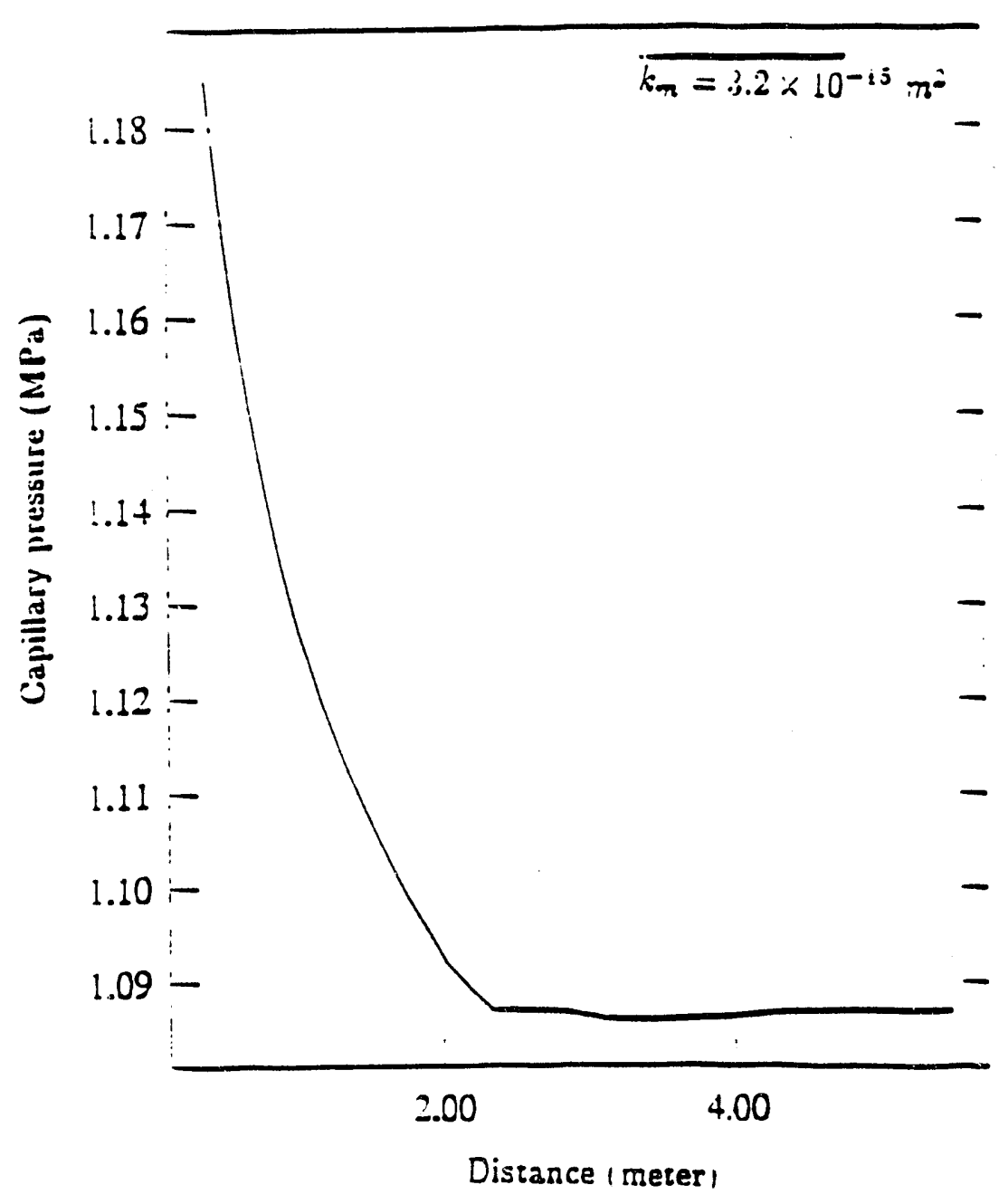

Figure 4(i): Capillary pressure profile at 1 year for $k_{m}=3.2 \times 10^{-15} \mathrm{~m}^{2}$ 


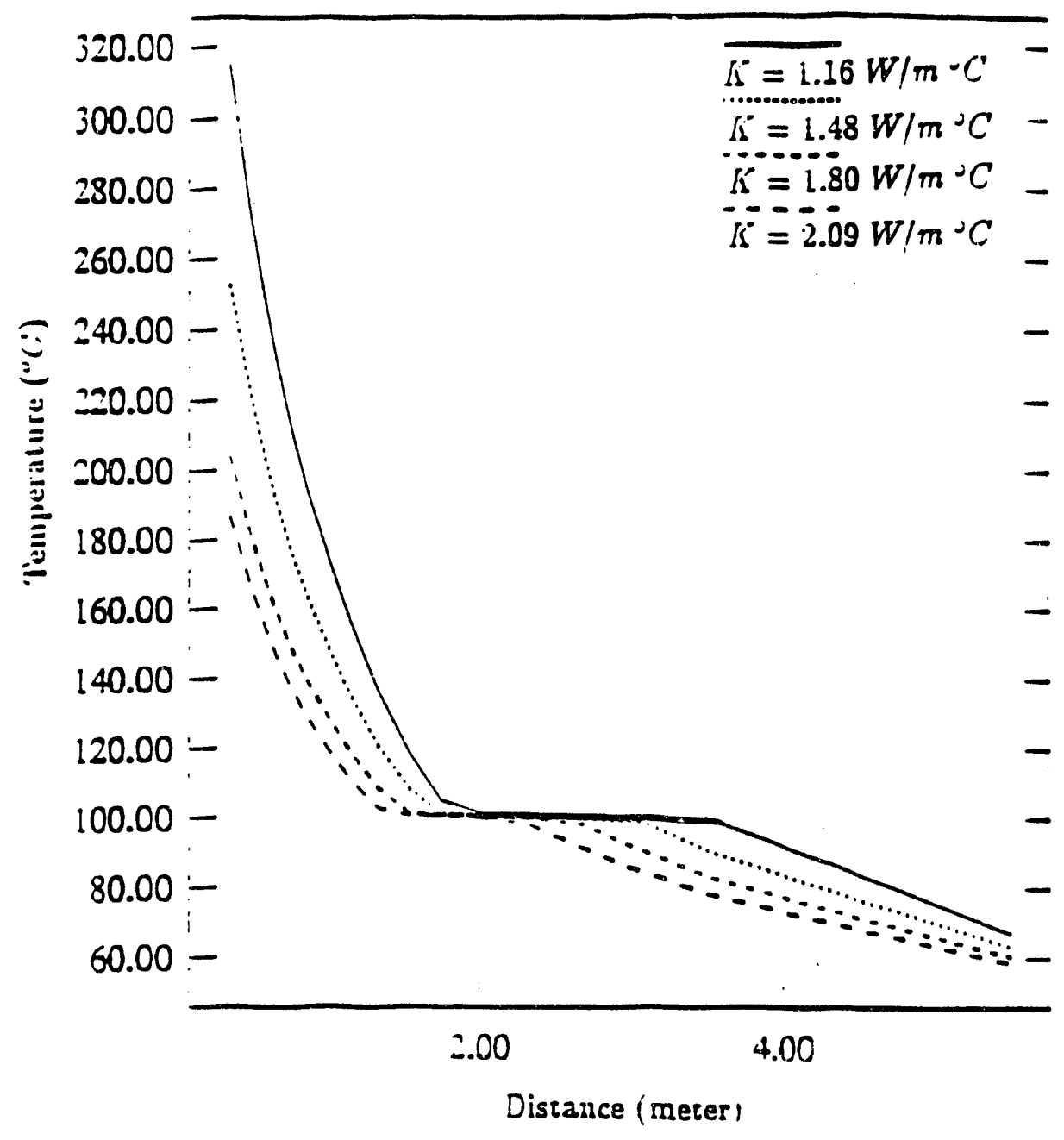

Figure $4(j)$ : Temperature proniles at 1 year for different thermal conductivities 


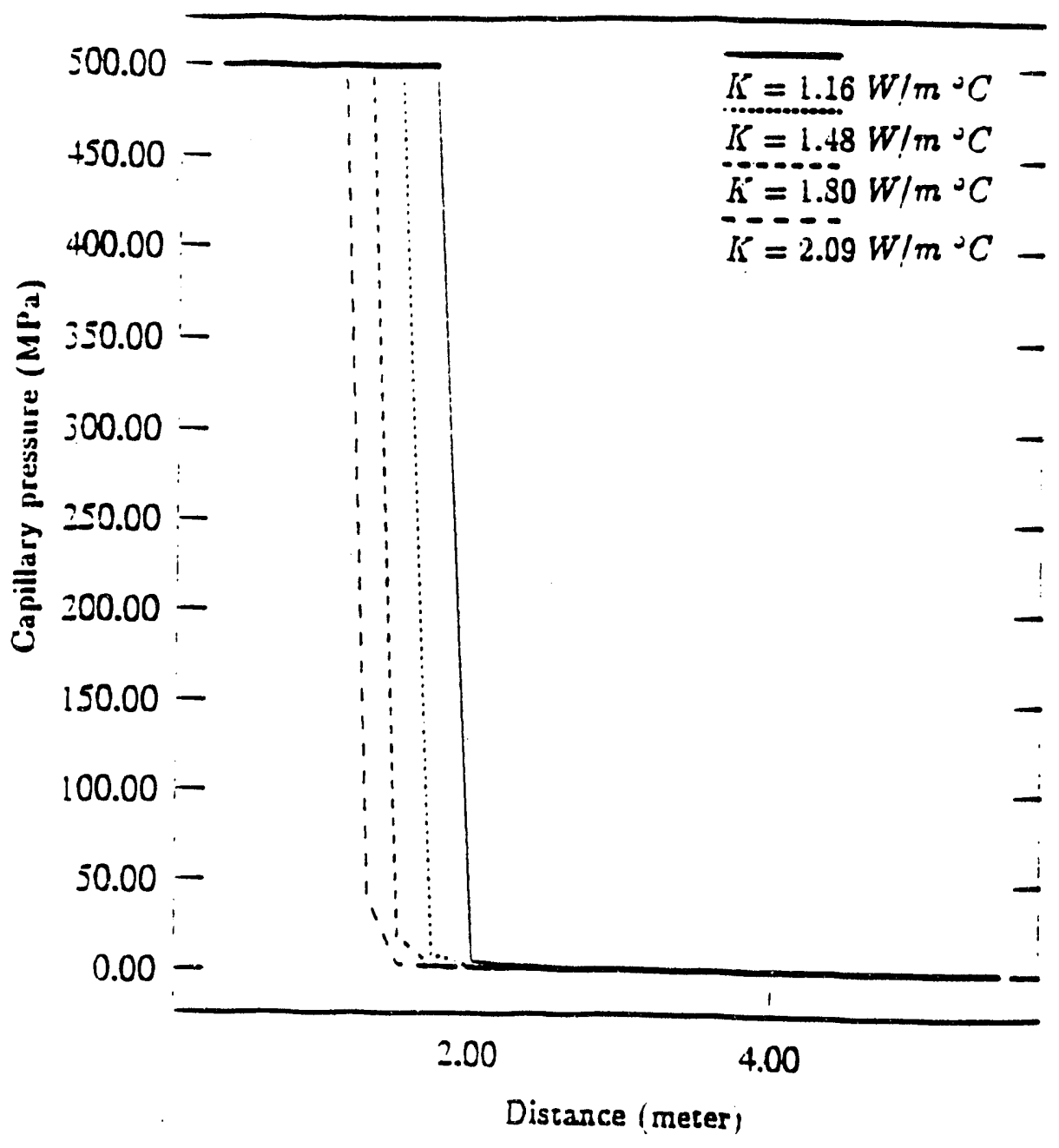

Figure 4(k): Capiilary pressure profiles at 1 year for different thermal conductivities 


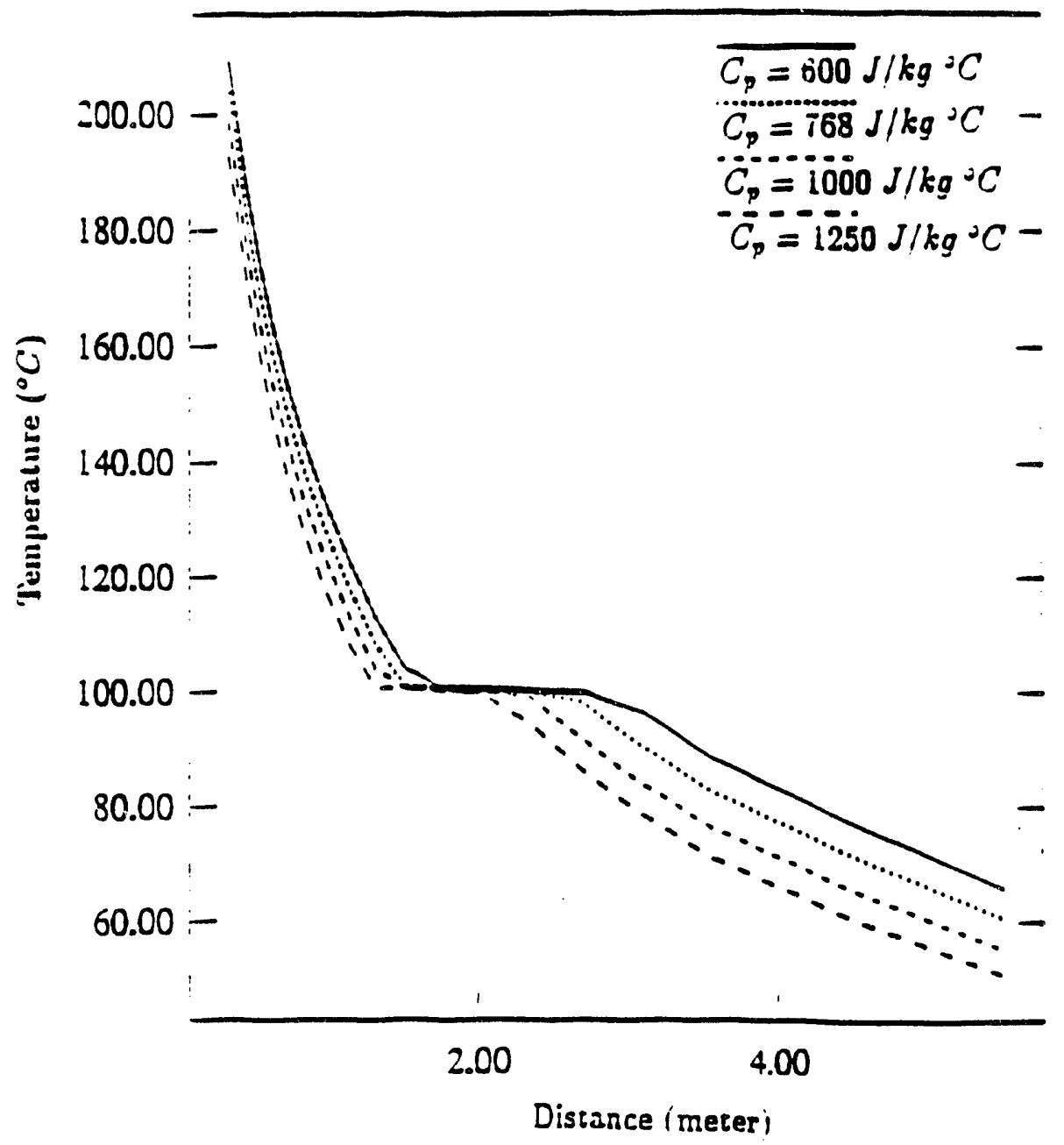

Figure $4(1)$ : Temperature proniles at 1 year for different specific heat 


\section{Chapter 5}

\section{EFFECT OF MASS INJECTION}

\subsection{INJECTION OF COLD WATER}

Simulations were carried cut to study the effect of liquid water injection on the performance of the heat pipe region in a repository. This would examine the effect of fracture flow toward the canister from some unspecified source. The reference values for the fracture and the matrix were used in all simulations. Water is injected in the fracture near the boundary away from the heat source. The permeability of the boundary element is made very small so that there is no liquid flow across the boundary and all the water flows in towards the heat source. The injection rates applied were $0.05 .0 .10,0.20,0.50$, and $1.0 \mathrm{~kg} / \mathrm{s}$ respectively. These rates correspond to some liquid velocities at the bore hole wall. These applied fluxes are also translated into some liquid velocities at the heat pipe region at a distance of 2.03 m from the cannister center line. The applied fluxes, the velocities at the bore hole wall and at the heat pipe region. and the pressure gradients to develop these velocities are given in table 5.1 . 
Table 5.1

\begin{tabular}{|l|l|l|l|l|}
\hline$q_{w}$ & $\begin{array}{l}V_{v, b} \\
(\mathrm{~kg} / \mathrm{s})\end{array}$ & $\begin{array}{l}V_{v, h} \\
(\mathrm{~m} / \mathrm{s})\end{array}$ & $\begin{array}{l}\left(\frac{d P}{d x}\right)_{b} \\
\left(\mathrm{~kg} / \mathrm{m}^{2} \mathrm{~s}^{2}\right)\end{array}$ & $\begin{array}{l}\left(\frac{d P}{d x}\right)_{h} \\
\left(\mathrm{~kg} / \mathrm{m}^{2} \mathrm{~s}^{2}\right)\end{array}$ \\
\hline 0.05 & $7.2 \times 10^{-4}$ & $9.6 \times 10^{-5}$ & $0.56 \times 10^{5}$ & $.75 \times 10^{4}$ \\
\hline 0.10 & $1.4 \times 10^{-3}$ & $1.9 \times 10^{-4}$ & $1.13 \times 10^{5}$ & $1.5 \times 10^{4}$ \\
\hline 0.20 & $2.9 \times 10^{-3}$ & $3.8 \times 10^{-4}$ & $2.25 \times 10^{5}$ & $3.0 \times 10^{4}$ \\
\hline 0.50 & $7.2 \times 10^{-3}$ & $9.6 \times 10^{-4}$ & $5.63 \times 10^{5}$ & $7.5 \times 10^{4}$ \\
\hline 1.0 & $1.4 \times 10^{-2}$ & $1.9 \times 10^{-3}$ & $11.2 \times 10^{5}$ & $15 \times 10^{4}$ \\
\hline
\end{tabular}

In table 5.1. $q_{w}, V_{w . b}, V_{w, h},\left(\frac{d P}{d x}\right)_{b}$, and $\left(\frac{d P}{d x}\right)_{h}$ represent the applied liquid flux. the liquid velocity at the bore hole wall, the liquid velocity at the heat pipe region. the pressure gradient at the bore hole. and the pressure gradient at the heat pipe respectively. Temperatures at selected distances for different injection rates are given in table 5.2 below.

Table $\mathbf{5 . 2}$

\begin{tabular}{|l|l|l|l|l|l|l|l|}
\hline \multirow{2}{*}{ Run } & \multirow{2}{*}{$\begin{array}{l}\text { Gw } \\
(\mathrm{kg} / \mathrm{s})\end{array}$} & \multicolumn{5}{|c|}{ Temperature in ${ }^{\circ} C$ at certain distances } \\
\cline { 3 - 8 } & $.27 \mathrm{~m}$ & $1.13 \mathrm{~m}$ & $1.52 \mathrm{~m}$ & $2.02 \mathrm{~m}$ & $2.33 \mathrm{~m}$ & $2.69 \mathrm{~m}$ \\
\hline 1 & 0.0 & 229.0 & 136.2 & 116.7 & 100.2 & 99.88 & 97.5 \\
\hline 2 & 0.05 & 229.4 & 136.6 & 117.2 & 101.3 & 100.9 & 97.2 \\
\hline 3 & 0.10 & 229.6 & 136.9 & 117.6 & 102.4 & 102.0 & 97.0 \\
\hline 4 & 0.20 & 228.9 & 136.3 & 117.2 & 104.8 & 104.1 & 96.7 \\
\hline 5 & 0.50 & 230.3 & 137.6 & 119.4 & 112.4 & 104.1 & 96.1 \\
\hline 6 & 1.0 & 233.5 & 1.45 .8 & 128.4 & 111.7 & 103.6 & 95.5 \\
\hline
\end{tabular}


The above results were compared with the simulation in which there is no liquid injection. The heat pipe region exists for the simuiation in which the liquid injection is zero. The jore hoie wall temperature decreases slightly with the increase in the injection rates. The iength of the heat pipe remains unchanged as the flux is increased, but higher temperatures are obtained in the heat pipe region and other points.

As the water injection rate is further increased, the heat pipe effect starts decreasing. The heat pipe length becomes smaller with the increase in injection rates, but the effect is gradual. The heat pipe region vanishes for $q_{w} \geq 0.50 \mathrm{~kg} / \mathrm{s}$. This flux corresponds to Lquid velocities of $7.2 \times 10^{-3} \mathrm{~m} / \mathrm{s}$ and $9.6 \times 10^{-4} \mathrm{~m} / \mathrm{s}$ at the bore hole wall and the heat pipe region respectively. Velocity, absolute permeability, dynamic viscosity and pressure gradient are related through the following equation.

$$
V=\frac{k}{\mu} \frac{d P}{d x}
$$

For water, the ratio $\frac{k}{\mu}$ is $1.28 \times 10^{-\gamma}$ so that the liquid velocity can be expressed by

$$
V_{w}=1.28 \times 10^{-8} \frac{d P}{d x}
$$

To achieve the above large velocities. very high pressure gradients are required. This situation may not arise in a practical case. which means the injection of liquid will not have a significant effect on the heat pipe performance. Fig. $5(a)$ shows all the temperature profiles at 1 year for different injection rates. From this graph. the gradual decrease of the heat pipe region with the increase in flow rates is easily understood. 


\subsection{INJECTION OF AIR}

The injection of air simulates the effect of air flow through a fracture system driven by pressure differential between the emplacement tunnel and the external environment. Air injection has the same effect as that of the liquid injection on the performance of the heat pipe. The air injection rate in $\mathrm{kg} / \mathrm{s}$ is denoted by $q_{a}$. The applied fluxes were $0.0001,0.0002$, $0.0003,0.0004$ and $0.0005 \mathrm{~kg} / \mathrm{s}$ respectively. The applied flux, the corresponding velocities at the bore hole wall and the heat pipe region, and the necessary pressure gradients to develop these velocities are given in table 5.3. $V_{a, b}, V_{a, h},\left(\frac{d P}{d x}\right)_{b}$, and $\left(\frac{d P}{d x}\right)_{h}$ represent the air velocity at the bore hole wall. air velocity at the heat pipe region. pressure gradient at. the bore hole and the pressure gradient at the heat pipe region at a distance of $2.03 \mathrm{~m}$ from the cannister center line respectively.

Table 5.3

\begin{tabular}{|l|l|l|l|l|}
\hline$q_{a}$ & $\begin{array}{l}V_{a, b} \\
(\mathrm{~kg} / \mathrm{s})\end{array}$ & $\begin{array}{l}V_{a . h} \\
(\mathrm{~m} / \mathrm{s})\end{array}$ & $\begin{array}{l}\left(\frac{d P}{d x}\right)_{b} \\
\left(\mathrm{~kg} / \mathrm{m}^{2} \mathrm{~s}^{2}\right.\end{array}$ & $\begin{array}{l}\left(\frac{d P}{d x}\right)_{h} \\
\left(\mathrm{~kg} / \mathrm{m}^{2} \mathrm{~s}^{2}\right)\end{array}$ \\
\hline .0001 & $1.2 \times 10^{-3}$ & $1.6 \times 10^{-4}$ & $.20 \times 10^{4}$ & $.27 \times 10^{3}$ \\
\hline .0002 & $2.4 \times 10^{-3}$ & $3.2 \times 10^{-4}$ & $.40 \times 10^{4}$ & $.54 \times 10^{3}$ \\
\hline .0003 & $3.6 \times 10^{-3}$ & $4.9 \times 10^{-4}$ & $.60 \times 10^{4}$ & $.81 \times 10^{3}$ \\
\hline .0004 & $4.8 \times 10^{-3}$ & $6.5 \times 10^{-4}$ & $.80 \times 10^{4}$ & $1.08 \times 10^{3}$ \\
\hline .0005 & $6.0 \times 10^{-3}$ & $8.2 \times 10^{-4}$ & $1.0 \times 10^{4}$ & $1.35 \times 10^{3}$ \\
\hline
\end{tabular}

The heat pipe region exists for the case in which there is no mass injection. Temperatures at selected distances for different injection rates after 1 year are given in table 5.4 below. 
Table 5.t

\begin{tabular}{|l|l|l|l|l|l|l|l|}
\hline \multirow{2}{*}{ Run } & $q_{\mathrm{a}}$ & \multicolumn{5}{|c|}{ Temperature in ${ }^{\circ} \mathrm{C}$ at certain distances } \\
\cline { 3 - 8 } & $\mathrm{kg} / \mathrm{s}$ & $.27 \mathrm{~m}$ & $1.13 \mathrm{~m}$ & $1.52 \mathrm{~m}$ & $2.02 \mathrm{~m}$ & $2.33 \mathrm{~m}$ & $2.69 \mathrm{~m}$ \\
\hline 1 & 0 & 220.0 & 136.2 & 116.7 & 100.2 & 99.88 & 97.5 \\
\hline 2 & .0001 & 229.6 & 136.8 & 117.5 & 102.0 & 101.6 & 99.1 \\
\hline 3 & .0002 & 229.2 & 136.6 & 117.3 & 103.7 & 103.2 & 99.0 \\
\hline 4 & .0003 & 228.7 & 136.0 & 117.0 & 105.3 & 104.7 & 96.8 \\
\hline 5 & .0004 & 228.5 & 135.8 & 116.8 & 107.0 & 105.0 & 96.7 \\
\hline 6 & .0005 & 229.0 & 136.2 & 117.0 & 108.5 & 105.0 & 96.8 \\
\hline
\end{tabular}

Fig.5(b) shows all the temperature profiles at 1 year for different air injection rates. All the results are compared with the standard case in which there is no air injection. The increase in air flux weakens the heat pipe. The heat pipe disappears for $q_{a} \geq .0005 \mathrm{~kg} / \mathrm{s}$. Further increase in air injection does not alter the temperature or liquid saturation considerably. As in the case of liquid injection. the disappearance of the heat pipe is gradual. $q_{a}=.0005$ corresponds to air velocities of $6.1 \times 10^{-3} \mathrm{~m} / \mathrm{s}$ and $8.2 \times 10^{-4} \mathrm{~m} / \mathrm{s}$ at the bore hole wall and the heat pipe region respectively. For air. the ratio $\frac{k}{\mu}$ is $0.59 \times 10^{-6}$ so that the air velocity can be expressed by the following equation.

$$
V_{a}=0.59 \times 10^{-6} \frac{d P}{d x}
$$

High pressure gradients are required to develop the above velocities which is quite uncommon in practical cases. so that the injection of air will not influence the heat pipe region in a repository. 


\subsection{WITHDRAWAL OF AIR}

The effect of air withdrawal is also tested for different withdrawal rates. Air is withdrawn from the bore hole wall. The same rates were used for both injection and the withdrawal. As the withdrawal rate increases. the temperature profile decreases. A withdrawal rate of $0.0002 \mathrm{~kg} / \mathrm{s}$ will cause the heat pipe effect to disappear. This withdrawal rate corresponds to an air velocity of $1.2 \times 10^{-3} \mathrm{~m} / \mathrm{s}$ at the bore hole wall and an air velocity of $1.6 \times 10^{-4} \mathrm{~m} / \mathrm{s}$ at the heat pipe region. Further increase in withdrawal reduces the temperature gradually. Table 5.5 gives the temperature distribution after 1 year for different withdrawal rates.

Table 5.5

\begin{tabular}{|l|l|l|l|l|l|l|l|}
\hline \multirow{2}{*}{$\begin{array}{l}\text { Run } \\
\#\end{array}$} & \multirow{2}{*}{$\begin{array}{l}q_{a} \\
\mathrm{~kg} / \mathrm{s}\end{array}$} & \multicolumn{5}{|c|}{ Temperature in ${ }^{\circ} \mathrm{C}$ at certain distances } \\
\cline { 3 - 8 } & $.27 .13 \mathrm{~m}$ & $1.52 \mathrm{~m}$ & $2.02 \mathrm{~m}$ & $2.33 \mathrm{~m}$ & $2.69 \mathrm{~m}$ \\
\hline 1 & 0 & 229.0 & 136.2 & 116.7 & 100.2 & 99.88 & 97.5 \\
\hline 2 & .0001 & 228.8 & 136.3 & 117.0 & 99.80 & 99.70 & 90.5 \\
\hline 3 & .0002 & 228.5 & 136.2 & 117.0 & 99.30 & 92.10 & 85.2 \\
\hline 4 & .0003 & 226.8 & 134.8 & 115.7 & 97.70 & 89.80 & 82.9 \\
\hline 5 & .0004 & 224.9 & 133.2 & 114.2 & 96.10 & 87.70 & 80.7 \\
\hline
\end{tabular}

From table 5.5 , it is clear that the temperature drops with the increase in withdrawal rate. The maximum pressure difference that can be achieved on a continuing basis with the air withdrawal is the atmospheric pressure. The maximum pressure gradient obtained in this case is not large enough to produce the high velocities calculated above. This again indicates that the withdrawal of air througn the bore hole will not change the heat pipe phenomenon in a repository. 
The effect of mass injection is not very important on the performance of the heat pipe in a repository. The injection of liquid water at the boundary reduces the heat pipe effect graduaily. The heat pipe effect is completely vanished for a liquid injection rate of $.05 \mathrm{~kg} / \mathrm{s}$. $A d r y$ out condition always exists in the near neld and is independent of the injection rate. This injection rate of $.05 \mathrm{~kg} / \mathrm{s}$ corresponds to a liquid velocity of $7.2 \times 10^{-3} \mathrm{~m} / \mathrm{s}$ at the bore hole wail. A very high pressure gradient is required to achieve this high velocity. In the case of air injection. the flux needed to change the heat pipe performance requires high velocities and large pressure gradients. These are very difficult to achieve in practical cases. 


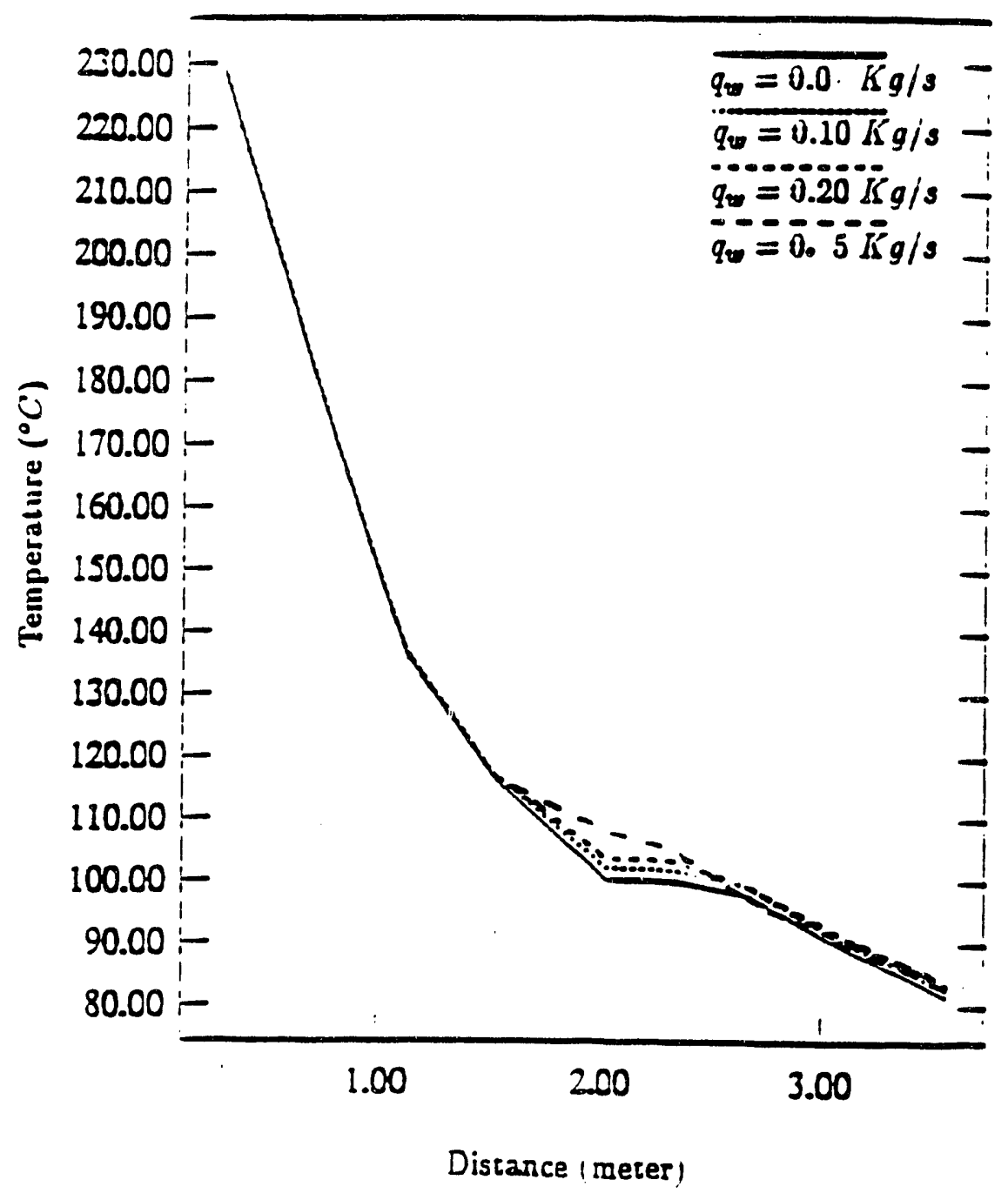

Figure $5(\mathrm{a})$ : Temperature proniles at 1 year for different water injection rates 


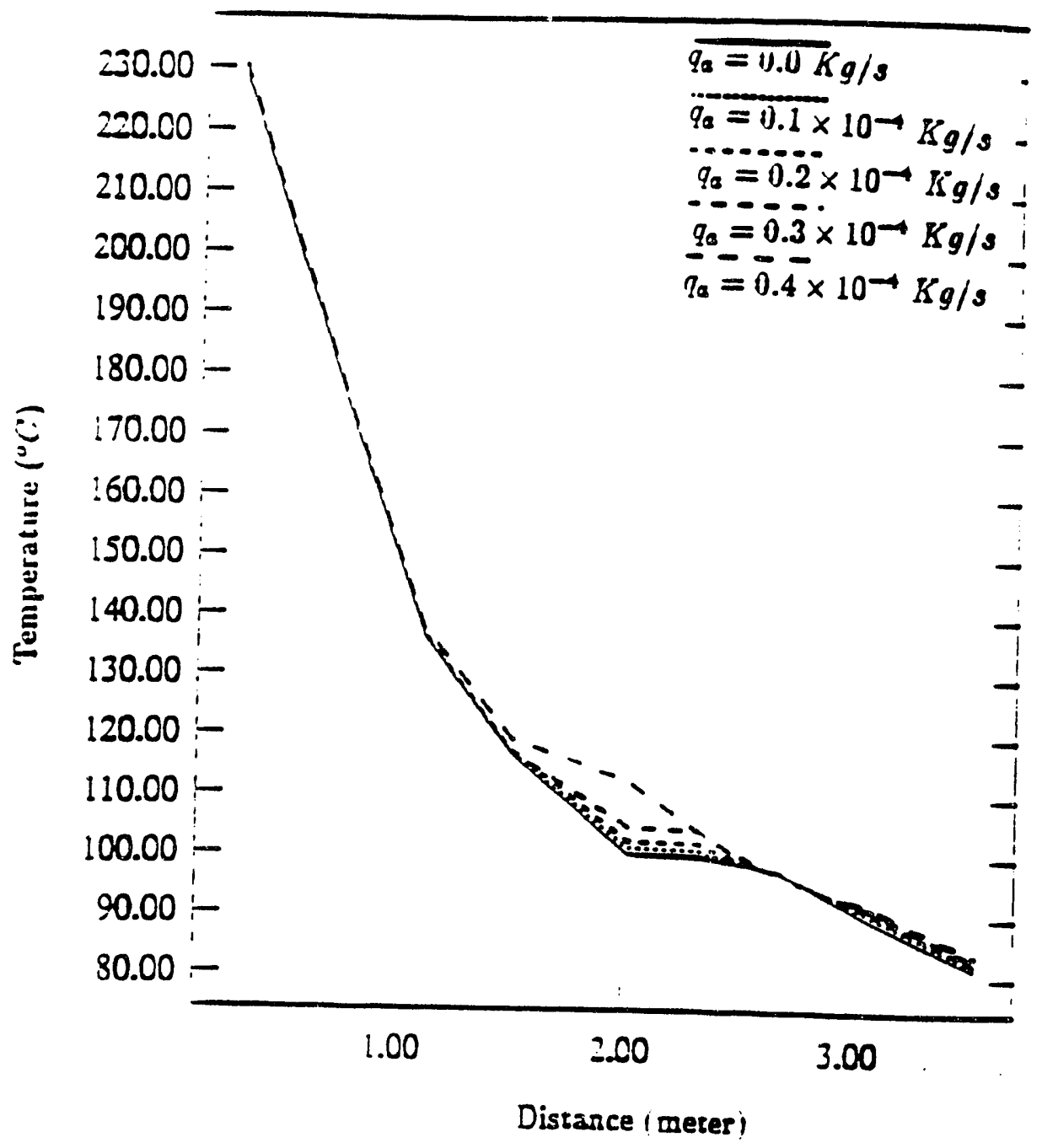

Figure $j(b)$ : Temperature proniles at 1 year for different air injection rates 


\section{Chapter 6}

\section{FRACTURE-MATRIX}

\section{EQUILIBRIUM}

Equilibrium between the fracture and the rock matrix in a repository is important in several ways. The development of the effective continuum approximation is based on the fracturematrix equilibrium. In a fractured medium. the fracture and the matrix are not always in equilibrium. In any case, some time is required for the fracture and the matrix to be in the thermodynamic equilibrium after the empiacement of nuclear waste cannisters in a repository. The parameters and conditions that may effect the final equilibrium are discussed in detail in this section. The equilibrium between the fracture and the outermost layer of the matrix is always considered to check the equilibrium between the fracture and the matrix. If they are in equilibrium, the entire matrix is considered to be in equilibrium with the fracture.

Permeability, thermal conductivity, porosity, speciñc heat and tortuosity may have some effect in the equilibrium. The most important parameters are the matrix permeability and 
the matrix thermal conductivity. The time required for the fracture-matrix equilibrium varies with the permeability and the thermal conductivity.

Permeability is the most important parameter that influences the fracture-matrix equilibrium. Large permeabilities reduce the time required for the equilibrium considerably. A large permeability provides an easy mean for the transport of heat and liquid so that the equiiibrium is achieved very quickly. In the case of small permeabilities, the interaction between the tight matrix and the fracture is very slow so that considerable time is needed for the fracture-matrix equilibrium.

Table 6.1 compares the time needed for the fracture and the matrix to be equilibrium at a distance of .97 meters from the center line of the cannister. This point corresponds to element $\# 9$ which is a common point in the heat pipe region for different permeabilities. The matrix is divided into three layers namely layer 1 . layer 2 and layer 3 . The distance from the fracture to the matrix increases with layer 1 , layer 2 and layer 3 respectively. If the fracture and laver 3 are in equilibrium. then the entire matrix will be in equilibrium with the fracture. $P_{c . f}-P_{c . m 3}$ represent the capillary pressure difference between the fracture and the outermost matrix laver(layer 3). As the permeability increases, this equilibrium time is reduced considerably. 
Table 6.1

\begin{tabular}{|l|l|l|l|l|l|l|}
\hline \multirow{2}{*}{$k_{m}$} & \multirow{2}{*}{$\left(m^{2}\right)$} & Time & \multicolumn{5}{|c|}{ Capillary pressure in different regions $\left(P_{a}\right)$} \\
\cline { 3 - 7 } & (days) & Fracture & layer 1 & layer 2 & layer 3 & $\left(P_{c, f}-P_{c, m 3}\right)$ \\
\hline $3.2 \times 10^{-18}$ & 51 & $.24 \times 10^{6}$ & $.25 \times 10^{6}$ & $.28 \times 10^{6}$ & $.39 \times 10^{9}$ & $.15 \times 10^{6}$ \\
\hline$"$ & 175 & $.50 \times 10^{9}$ & $.50 \times 10^{9}$ & $.50 \times 10^{9}$ & $.35 \times 10^{8}$ & $.465 \times 10^{9}$ \\
\hline$"$ & 265 & $.50 \times 10^{9}$ & $.50 \times 10^{9}$ & $.50 \times 10^{9}$ & $.50 \times 10^{9}$ & 0 \\
\hline $3.2 \times 10^{-17}$ & .51 & $.85 \times 10^{6}$ & $.86 \times 10^{6}$ & $.87 \times 10^{6}$ & $.92 \times 10^{6}$ & $.07 \times 10^{6}$ \\
\hline$"$ & .55 & $.50 \times 10^{9}$ & $.50 \times 10^{9}$ & $.50 \times 10^{9}$ & $.50 \times 10^{9}$ & 0 \\
\hline $3.2 \times 10^{-16}$ & .51 & $.107 \times 10^{7}$ & $.107 \times 10^{7}$ & $.107 \times 10^{7}$ & $.108 \times 10^{7}$ & $.10 \times 10^{5}$ \\
\hline$n$ & 65 & $.111 \times 10^{7}$ & $.111 \times 10^{7}$ & $.111 \times 10^{7}$ & $.111 \times 10^{7}$ & 0 \\
\hline $3.2 \times 10^{-15}$ & 39 & $.109 \times 10^{7}$ & $.109 \times 10^{7}$ & $.109 \times 10^{7}$ & $.109 \times 10^{7}$ & 0 \\
\hline
\end{tabular}

Table 6.1 shows how the equilibrium time changes with matrix permeability. $\left(P_{c, f}-\right.$ $\left.P_{c, m 3}\right)=0$ means the equilibrium between the fracture and layer 3 and this indicates the fracture-matrix equilibrium. The minimum permeability of $3.2 \times 10^{-18} \mathrm{~m}^{2}$ needed 265 days for the fracture-matrix equilibrium. The maximum permeability of $3.2 \times 10^{-15} \mathrm{~m}^{2}$ required just 39 days for the equilibrium at the same point in the heat pipe region. This shows the significance of the permeability.

Thermal conductivity also affects the equilibrium time. The equilibrium time for the thermal conductivity was also compared at a common point in the heat pipe region(.97 m). Higher thermal conductivities require less time to achieve equilibrium compared to the smaller thermal conductivities. Compared to the permeability, thermal conductivity produced only minor changes in equilibrium time. For higher thermal conductivities, the 
temperature in the near region around the cannister decreases. A maximum temperature of $138^{\circ} \mathrm{C}$ was observed at the bore hole wail for the maximum thermal conductivity of 2.09 used for the simulations. The corresponding temperature for the minimum thermal conductivity of 1.16 was $211^{\circ} \mathrm{C}$.

The time required for the fracture-matrix equilibrium does not vary with change in the porosity. Specific heat and tortuosity also do not affect the equilibrium time. 


\section{Chapter 7}

\section{CONCLUSIONS \&}

\section{RECOMMENDATIONS}

The mathematical modeling of the heat pipe effect produced by a thermal source in fractured porous media generated some very interesting results. The issues focused in this study include the effect of fracture and matrix properties on the behavior of the heat pipe, the importance of convective heat transfer over conductive heat transfer in heat pipe region, the effect of mass injection and mass withdrawal on heat pipe, and the factors affecting the fracture-matrix equilibrium.

The fracture parameters namely the permeability, the thermal conductivity, the specific heat, the porosity, and the tortuosity do not change the heat pipe performance, but the mobility of the liquid in the fracture changes the heat pipe performance very significantly. This was previousiy shown by [Pruess et al.. 1990]. For the mobile case, the heat pipe region is extended up to the heat source and the near region surrounding the cannister remains in a two phase condition. This increases the mass transport and brings the moisture into the 
vicinity of the cannister. The type of relative permeability and capillary pressure functions in the fracture do not change the results either in the mobile case or in the immobile case. The key issue here is the mobility or the immobility of the liquid in the fracture.

Some of the matrix properties influence the heat pipe phenomenon in the repository. Matrix permeability and matrix thermal conductivity are the two most important properties. Larger permeabilities increase the heat pipe length and extend the the heat pipe region up to the bore hole wail. producing two phase conditions in the region surrounding the bore hole wall with temperatures remaining at the boiling temperature which is near $100^{\circ} \mathrm{C}$. A dry out condition is not achieved in this case. An increase in thermal conductivity also reduces the temperatures and shifts the heat pipe region towards the cannister. The matrix specific heat. the porosity, and the tortuosity do not have considerable effect on the heat pipe region.

Convection is a measure of the strength of the heat pipe. A heat pipe is said to be stronger if the convection dominates over the conduction in the heat pipe region. The net flow of heat in the matrix is towards the cannister. The net flow of heat in the fracture is due to the convection and it is always away from the cannister. The net flow of heat in the overall system is away from the heat source. For higher permeabilities, strong convective effects are observed and the mass transfer is increased.

Injection of water and air through the boundary towards the cannister produced the gradual disappearance of the heat pipe with increasing flux. These fluxes correspond to high velocities at the bore hole wall and at the heat pipe region. High pressure gradients are required to develop these velocities and this is probably not achievable in practical cases. The withdrawal of air from the bore hole wall produced the sudden disappearance of the 
heat pipe, but again this flux requires a high pressure gradient to develop the corresponding velocity. This situation is very difficult to achieve in normal cases.

An equilibrium between the fracture and the matrix is developed some time after the emplacement of the cannisters. The most important parameters affecting the equilibrium are the absolute permeability and the thermal conductivity of the matrix. Large permeabilities provide an easy means for the transport of the heat and the liquid and reduce the equilibrium time. An increase in thermal conductivity reduces the time needed for the fracture-matrix equilibrium. The porosity, the specific heat and the tortuosity do not affect the equilibrium time.

Future work can be done in several areas. The capillary pressure and relative permeability functions for the fracture are not fully available at present. These parameters do change the flow pattern significantly. The presence of expected vertical fractures are not accounted in this work.

The present work is done for one-dimensional radial configuration. The existing model can be extended to a large scale by developing a 3-dimensional model. The heat pipe may provide a mechanism for the contarninant transport under favourable conditions. It will be very interesting to observe the effect of the heat pipe on the movement of the contaminant and other radioactive particles. 


\section{BIBLIOGRAPHY}

1. Bear. J., Zaslavsky, D.. and Irmay, S., *Physical principles of water percolation and seepage," June. 1968.

2. Bixler, N.A., "NORIA - A finite element computer program for analyzing water, vapor, and energy transport in porous media." SAND84-2057, Sandia National Laboratories, Albuquerque NM. August, 1985.

3. Doughty, Christine. and Pruess. K.. "A semianalytical solution for heat pipe effects near high-level nuclear waste packages buried in partially saturated geological media." Int. J. Heat Mass transfer. 31(1), 79-90. 1987.

4. Duff, I.S., "MA28 - A finite element computer program for analyzing water, vapor, and energy transport in porous media." Report AERE $R$ 8730, U.K. Atomic Energy Authority, Harwell Laboratories. United Kingdom. 1977.

5. Fitch. J.S. and Udell. K.S.. "Limits of multiphase heat and mass transfer in porous media." ALAA/ASME Thermophysics and Heat Transfer Conference, Boston. MA. June, 1986.

6. Genuchten. V., "A closed form equation for predicting the hydraulic conductivity of unsaturated soils," Soil Sci. Soc. Am. J.. 44, 892-898, 1980.

7. Hadley, G.R., "PETROS - A program for calculating heat, water, water vapor, and air through a porous material." SAND84-0878. Sandia National Laboratories. Albuquerque NM. May, 1985. fluid flow in fractured porous media." Water Resources 
Research. 18(4), 1235-1247. 1982.

8. Nitao. J.J. and Bushcheck. T.A.. "On the infiltration of a liquid front in an unsaturated. fractured porous medium," Rep. C'CRL-100777, Lawrence Livermore National Laboratory, August. 1089.

9. Ogniewicz. Y. and Tien, C.L.. "Porous heat pipe," AIAA Thermophysics Conference, Orlando. Fla., 1979.

10. Peters. R.R.. Klavetter. E.A.. Hall. I.J.. Blair. S.C.. Heller. P.R. and Gee, G.W., "Fracture and matrix hydrologic characteristics of tuffaceous materials from Yucca mountain. Nye County, Nevada," Rep. SAND 84-1471, Sandia Natl. Lab., Dec., 1984.

11. Pruess. K.. "Tough user's guide," SAND 21-5303. Sandia Natl. Lab., June, 1987.

12. Pruess. K.. "A quantitative model of vapor dominated geothermal reservoirs as heat pipes in fractured porous rock," Geothermal Resources Council, Trans., 9(2), 353-359, 1985.

13. Pruess, $K$.. "Modeling studies of multiphase fluid and heat flow processes in nuclear waste isolation." .Hat. Res. Soc. Symp. Proc.. 127, 793-803, 1989.

14. Pruess. K.. Wang, J.S.Y. and Tsang, Y.W., "On thermohydrologic conditions near high-level nuclear wastes emplaced in partially saturated fractured tuff - Part I," Water Resources Researci, 26(6), 1235-1248, 1990. 
15. Pruess. K., Wang. J.S.Y. and Tsang, Y.W.. "On thermohydrologic conditions near high-level nuclear wastes emplaced in partially saturated fractured tuff - Part II." Water Resources Research. 26(6), 1249-1261. 1990.

16. Reddy, G.B.. "Anaiysis of heat and mass transfer in unsaturated porous materials: An application to soil.” Ph.D. Dissertation. North Carolina State University, Raleigh, August 1986.

17. Somerton. W.H.. Keese. J.A.. and Chu. S.L.. "Thermal behavior of unconsolidated oil sands." paper SPE-4506. 48th annual fall meeting of the society of petroleum engineers. Las Viegas, NV. 1973.

18. Udell. K.S., "Heat transier in porous media considering phase change and capillaritythe heat pipe effect." Int. J. Heat Mass Transfer, 28(2), 485-495, 1985.

19. Udell. K.S. and Fitch. J.S., "Heat and mass transfer in capillary porous media considering evaporation. condensation and non-condensible gas effects," $A S M E / A I C h E$ National Heat Transfer Conference, Denver, CO, August, 1985.

20. Updegraff, C.D.. "Comparison of Strongly heat-driven flow codes for unsaturated media." SAND 38-7145, Sandia National Laboratories. August, 1989.

21. Wang, J.S.Y. and Narasimhan. T.N., "Hydrologic mechanisms governing fluid flow in a partially saturated, fractured, porous medium." Water Resources Research, 21(12), $1861-1874,1985$. 

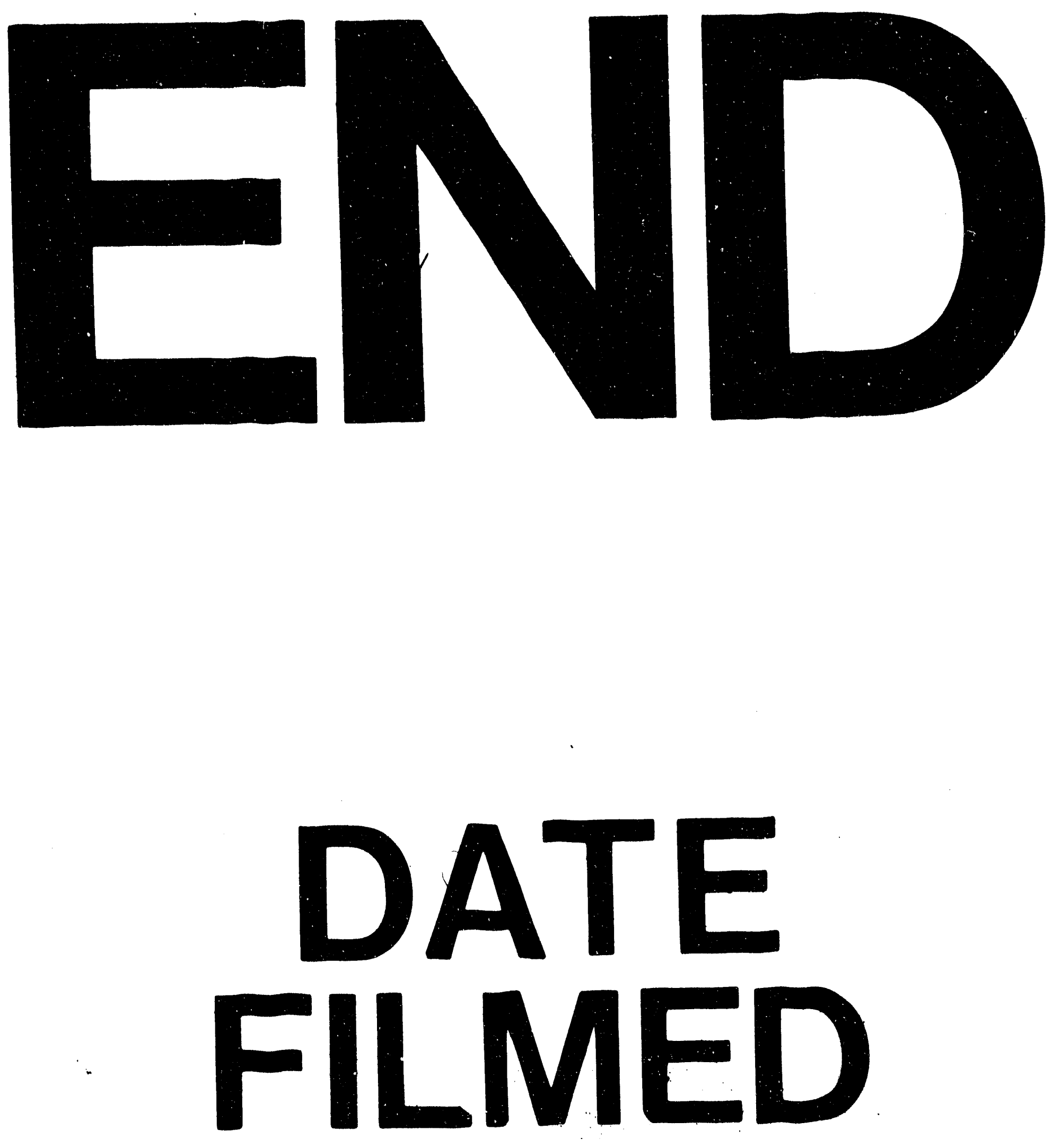

1

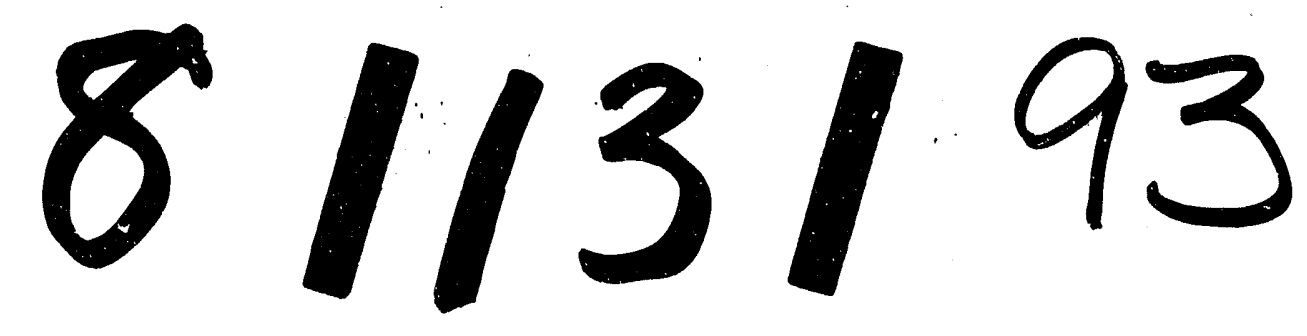

